\title{
Analyse retard des mesures d'auscultation de barrages
}

\section{S. BONELLI}

Cemagref

Unité de recherche

Ouvrages hydrauliques et équipements pourl'irrigation BP 31

13612 Aix-en-Provence Cedex 1

stephane.bonelli@ cemagref.fr

Laboratoire de Mécanique et d'acoustique, UPR CNRS 7051

31, chemin Joseph-Aiguier 13402 Marseille
L'auscultation des barrages fournit des séries chronologiques de mesures qui doivent être analysées. Pour comprendre le comportement hydraulique de l'ouvrage, il est essentiel de représenter les effets différés dus à la diffusion en milieu poreux. Un modèle permettant une analyse retard des mesures de pression interstitielle est présenté. Il est basé sur une représentation approchée de la réponse impulsionnelle de l'ouvrage permettant de reconstituer les variations mesurées qui sont provoquées par le niveau de la retenue et par la pluie. Après une analyse théorique, le modèle est comparé à des solutions exactes d'un problème de diffusion linéaire, puis appliqué à l'analyse de quelques mesures d'auscultation de trois barrages.

Mots-clés : milieu poreux, pression interstitielle, effet différé, réponse impulsionnelle, barrage, auscultation, série chronologique, ARMA.

\section{Delayed response analysis of dam monitoring data}

Long series of monitoring data are obtained during the routine operation of a dam. To understand the long term behaviour of a dam, it is essential to carefully interpret the dissipative effects which tend to occur due to seepage, in order to be able to distinquish between the effects of factors such as drift, irreversible events and the ageing of the dam and the effects of other factors not involving ageing processes. A model for performing delay analysis on pore pressure measurements is presented. The method involves the use of an approximate impulse response accounting for the contribution of non-ageing factors, i.e., the reservoir level and rainfall events, to the pore-pressure variations. Following theoretical analysis, the model is compared with closed-form solution of a diffusion problem, and finally applied to three dams studies.

Key words : porous media, pore pressure, delayed effect, impulse response, dam, monitoring, time series, ARMA. 


\section{Introduction}

Les méthodes statistiques de type Hydrostatique-Saison-Temps (également appelées Hydrostatic-SeasonTime ou HST) ont été proposées dans les années 60 pour analyser les mesures de déplacements issues de pendules de barrages voutes (Ferry et Willm, 1958; Willm et Beaujoint, 1967; Lugiez et al., 1970). Ces méthodes sont actuellement utilisées dans plusieurs pays, pour analyser d'autres types de mesures (Guedes et Coehlo, 1985; Silva Gomes et Silva Matos, 1985; Crépon et Lino, 1999; Carrère et al., 2000). L'expérience acquise depuis quelques décennies sur plusieurs centaines de barrages a confirmé l'excellence de l'approche comme un outil puissant d'interprétation des mesures d'auscultation. La littérature ne fait toutefois pas état d'une méthode opérationnelle tenant compte des effets différés, qui permettrait d'analyser les mesures hydrauliques, influencées par le niveau de la retenue et par la pluie.

Les effets différés sont dus à un comportement dissipatif : l'écoulement en milieu poreux. La nécessité de considérer ces effets est bien connue (Lugiez et al., 1970). La prise en compte de la réponse impulsionnelle d'un milieu poreux semi-infini a permis quelques analyses retard de I'influence de la retenue sur des cellules de pression interstitielle dans un noyau de barrage (Poupart, 1994), mais l'analyse et son interprétation restent délicates. Cet article propose un modèle retard destiné à reconstituer les pressions interstitielle mesurées dans les barrages ou ses abords, et influencées par la retenue ou par la pluie. Ce modèle est basé sur la réponse impulsionnelle de l'ouvrage (également appelèe Impulse-Response-Function ou IRF).

La première partie analyse les lacunes de l'approche classique. Elle démontre la nécessité de prendre en compte les effets différés lors de l'analyse des mesures de pression interstitielle. La deuxième partie est consacrée à la représentation externe d'un problème parabolique linéaire: représentation exacte à partir de la fonction de Green et représentation approchée à partir d'un modèle retard exponentiel (exponential-IRF). Une solution exacte originale bidimensionnelle, présentée dans la troisième partie, permet de valider l'approche et de donner une signification mécanique aux paramètres du modèle. L'application à l'analyse de quelques mesures d'auscultation de trois barrages est présentée dans la dernière partie.

\section{2}

\section{Nécessité d'une analyse retard}

\section{1}

\section{Lacunes du modèle Hydrostatic-Season-Time}

Le modèle HST est basé sur trois effets. Le premier est l'effet hydrostatique, qui rend compte des variations $H$ de la mesure provoquées par la retenue. Il est représenté par un polynôme - souvent d'ordre quatre du niveau de la retenue à l'instant $t$ :

$$
\begin{gathered}
H(t)=b_{1} z(t)+b_{2} z^{2}(t)+b_{3} z^{3}(t)+b_{4} z^{4}(t) . \\
z(t)=\frac{Z(t)-Z_{\min }}{Z_{\max }-Z_{\min }}
\end{gathered}
$$

où $Z_{\text {min }}$ est un niveau minimal (cote du tapis drainant par exemple) et $Z_{\text {max }}$ un niveau maximum (côte de la crête, ou cote d'exploitation normale par exemple). Le deuxième effet est la date dans l'année, qui rend compte des variations saisonnières $S$ de la mesure, de périodes douze mois et six mois. Il est représenté par les deux premiers termes d'un développement en série de Fourier:

$$
S(t)=A_{1} \sin \left(\omega_{2}\left(t+d_{1}\right)\right)+A_{2} \sin \left(2 \omega_{n}\left(t+d_{2}\right)\right), \omega_{n}=\frac{2 \pi}{\Delta T_{u}}(2)
$$

où $\omega_{\alpha}$ est la pulsation annuelle ( $\Delta T_{\text {correspond }}$ à un an). Le troisième effet rend compte de l'influence du temps, dont le vieillissement. Son expression est variable, et dépend du phénomène étudié. Cet effet est souvent dénommé "effet irréversible ».

Cette approche est classique en analyse de données. Elle est utilisée dans de nombreux autres domaines (Young, 1998). L'un des plus anciens exemples connus est l'ajustement sinusoildal de Forbes (1846) pour reproduire les variations cycliques de la température du sol. Pour les barrages, l'approche est robuste et conduit dans la plupart des cas à des résultats convenables. Elle comporte toutefois deux lacunes : 1) ses paramètres ont peu de sens mécanique; 2) elle ne tient pas compte de la structure particulière des séries chronologiques analysées.

L'expression polynomiale de l'influence de la retenue est historique. Elle est issue de la résistance des matériaux pour analyser des déplacements : l'influence de la pression hydrostatique sur les déplacements amont/aval d'un barrage voûte. Cette variable explicative est souvent utilisée par défaut pour l'analyse des mesures hydrauliques, mais une relation polynomiale de degré quatre (ou plus) entre un niveau piézométrique et le niveau de la retenue au même instant n'est pas mécaniquement justifiée.

Les effets saisonniers sont bien connus sur les barrages voûtes. Ils correspondent à l'influence des écarts de température entre les saisons froides et les saisons chaudes sur les déplacements mesurés. Pour les mesures hydrauliques, cet effet est également constaté, notamment sur les barrages en terre destinés à l'alimentation en eau ou à l'irrigation. Lorsque l'exploitation de la retenue est saisonnière, la composante hydrostatique et la composante saisonnière sont fortement corrélées, Dans ce cas, l'analyse statistique et son interprétation deviennent délicates.

La prise en compte de la pluie est nécessaire pour analyser les mesures hydrauliques. Elle concerne tous les barrages: les abords sont toujours auscultés par des piézomètres. Une méthode simple est de prendre la pluie cumulée sur les dix derniers jours (Crépon et Lino, 1999). La prise en compte de cumuls sur plusieurs périodes antérieures peut conduire à de bons résultats (Bonelli et al., 1998), mais l'approche demeure purement statistique.

\section{2}

\section{Exemples de mesure avec effet retard}

La figure 1 illustre un exemple de mesure piézométrique sur la plate-forme en aval d'un barrage. A priori, les variations des premières années sont proportionnelles au niveau de la retenue (Fig. 1a), ce qui devrait se traduire par une relation linéaire entre variation piézométrique et variation de la retenue. Il n'en est rien (Fig 1b), et un polynôme du type (1), mème d'ordre 

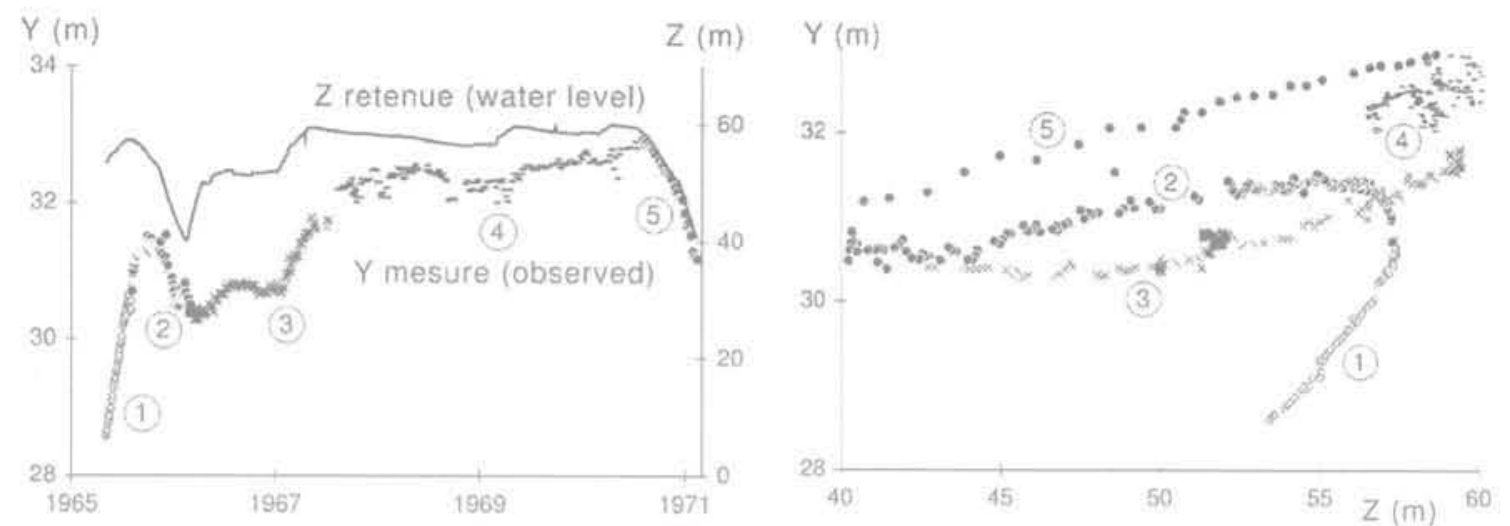

FiG. 1 Esemple de mesure piézométrique, niveaux piézométriques en fonction du temps et du niveau de la retenue, hystérésis d'un cycle de hausse/diminution de la retenue.

Example of piezometer data, piezometric head vs. time, piezometric head vs. water level, hysteresis of a cyclic rise/drop in the water level.

élevé, n’en rendrait pas compte. De plus, si la périodicité des mesures est indépendante des variations de la retenue (ce qui est souvent le cas en pratique), ces variations pourront être statistiquement et accidentellement bien expliquées par le modèle instantané polynomial (1), sans aucun fondement mécanique. En effet. lorsque les diminutions sont lentes du fait de l'exploitation, les mesures sont nombreuses. Lorsque les hausses sont rapides du fait de précipitations importantes, les mesures sont rares. L'ajustement sera alors réalisé sur les mesures les plus nombreuses, et le résultat ne sera pas interprétable.

La figure 1 montre qu'un cycle de hausse/diminution du niveau de la retenue est dissipatif (hystérésis) : le trajet n'est pas le même pour une hausse (phase 3) et pour une diminution (phase 5). Certaines mesures peuvent indiquer une augmentation de pression interstitielle pendant que le niveau du réservoir décroit, et inversement. Ce phénomène bien connu est dû à la capacité d'emmagasinement : variation de porosité ou présence d'air. Il est constaté sur ouvrage (Kjaernsli et al., 1982; Myrvoll et al., 1985) et a été reproduit en laboratoire (Windish et Høeg, 2000). Cet exemple illustre ce qu'est l'effet retard et le fait que le modèle (1) ne peut en rendre compte. Il sera repris ultérieurement.

\section{3}

\section{Nécessité d'une description externe}

Les analyses des mesures d'auscultation traitent un grand nombre de données et constituent un élément de décision pour évaluer la sécurité de l'ouvrage. Il est fondamental qu'elles soient faites périodiquement, dans un délai court. Afin de quantifier les évolutions à conditions constantes (dont le vieillissement), il faut au préalable être capable de rendre compte des évolutions indépendantes du temps, uniquement dues aux sollicitations extérieures (variations de la retenue et précipitations). La description interne par variables d'état conduit à des modèles à grand nombre d'inconnues. souvent basés sur la méthode des volumes finis ou des éléments finis, Cette approche est réservée aux grands ouvrages, Pour les abords (rives, appuis, plate-forme aval) également auscultés, de telles modélisations sont mal adaptées : il est difficile - voire impossible - d'accéder aux caractéristiques géométriques et matérielles.

Une description externe basée sur les réponses impulsionnelles ne nécessite aucune connaissance préalable de la géométrie du barrage ni des propriétés des matériaux. Les ouvrages spécialisés en traitement du signal et en automatique contiennent les bases des méthodes et leurs résultats (Roïtenberg, 1974; Faure et Robin, 1984 ; Santamaria et Fratta, 1998). Nous utiliserons une description basée sur la réponse impulsionnelle de l'ouvrage (Impulse-Response-Function ou IRF).

L'utilisation de réponses impulsionnelles dans un cadre linéaire doit permettre de vérifier quelques propriétés. Le modèle doit être stationnaire (invariant par translation de l'origine des temps). Le temps n'a qu'une dimension cinématique caractéristique des phènomènes dissipatifs (au sens de l'enchainement des événements à une certaine vitesse), mais pas de dimension géologique (au sens où l'origine des temps, donc l'âge du systeme, n'intervient pas): La quantification des effets stationnaires est une question fondamentale de l'analyse des mesures d'auscultation (Fanelli et al., 2000). Le terme anon stationnaire $)$ nous semble plus pertinent que le terme "irréversible » pour qualifier les effets du temps : les phénomènes dissipatifs comme l'écoulement en milieu poreux peuvent correspondre à une évolution stationnaire et irréversible. Ce caractère dissipatif impose de considérer l'histoire des sollicitations pour expliquer les niveaux à un instant clonné, et non la seule valeur de ces sollicitations au même instant. Par ailleurs, un phénomène essentiel doit être reproduit : l'accommodation. La réponse en régime permanent à un signal sinusoïdal (essai harmonique) doit être un signal sinusoïdal (accommodation) de mème période, déphasé et d'amplitude différente. L'accommodation est exactement ce que vise à modéliser la variable saisonnière du modèle Hydrostatic-Season-Tïme. On peut s'attendre à ce qu'une variable explicative décrite par une réponse impulsionnelle constitue une avancée pour reproduire les variations saisonnières des mesures hydrauliques. 


\section{Représentation externe d'un problème parabolique}

\section{1}

\section{Représentation externe exacte}

Par souci de simplicité, nous parlerons indiffẻremment de pression interstitielle, de charge hydraulique ou de niveau piézométrique. On considère le problème parabolique linéaire anisotrope suivant :

$$
\begin{gathered}
c \frac{\partial p}{\partial t}(r, t)-\nabla \cdot(\underline{K} \cdot \nabla p(r, t)=0 \text { dans } \Omega, \\
p(r, 0)=p_{0}(r) \text { dans } \Omega, \\
p(r, t)=p_{1}(t) \text { sur } \Gamma_{1},-\underline{n} \cdot \underline{K} \cdot \nabla p(r, t)=q(t) \operatorname{sur} \Gamma_{2}, \\
(r, t)=0 \text { sur } \Gamma_{3},-\underline{n} \cdot \underline{K} \cdot \nabla p(r, t)=0 \text { sur } \Gamma_{4}
\end{gathered}
$$

où $p(r, t)$ est la charge hydraulique, fonction des coordonnées spatiales $r$ et du temps $t, \Omega$ est un milieu poreux de frontière $\Gamma=\Gamma \cup \Gamma_{2} \cup \Gamma_{3} \cup \Gamma_{4}$, de capacité cet de conductivité hydraulique anisotrope $\underline{K} \underline{\underline{n}}$ étant la normale unitaire extérieure à $\Gamma$. On suppose pour simplifier que la condition initiale $(3 \mathrm{~b})$ vérifie les conditions aux limites $(3 \mathrm{c}, \mathrm{d})$ à l'instant initial. Cette hypothèse ne nuit pas à la généralité des présents raisonnements : si tel n'était pas le cas, d'autres termes se rajouteraient à la description. Une représentation de la solution du problème (3) peut être déduite de résultats classiques (Roach, 1970; Beck et al., 1992):

$p(r, t)=p_{0}(r)+h_{0}\left(r, t, p_{0}\right) * u(t)+h_{1}(r, t) * p_{1}(t)+h_{2}(r, t) * q(t)$

dans $\Omega$

avec

$$
\begin{gathered}
h_{0}\left(r, t, p_{0}\right)=-\frac{1}{c} \int g\left(r, r^{\prime}, t\right) \nabla_{r} \cdot\left(K \cdot \nabla_{r} \cdot p_{0}\left(r^{\prime}\right)\right) d r^{\prime}, \\
h_{1}(r, t)=-\frac{1}{c} \int \underline{n}\left(r^{\prime}\right) \cdot \underline{K} \cdot \nabla_{r^{\prime}} g\left(r, r^{\prime}, t\right) d r^{\prime}, \\
h_{2}(r, t)=-\frac{1}{c} \int g\left(r, r^{\prime}, t\right) d r^{\prime}
\end{gathered}
$$

où $g\left(r, r^{\prime}, t\right)$ est la fonction de Green associée au problème (3). L'opérateur $(\alpha+)$ est le produit de convolution temporelle défini sur des grandeurs causales:

$$
(h * a)(t)=\int_{0}^{t} h\left(t-t^{\prime}\right) a\left(t^{\prime}\right) d t^{\prime}=\int_{0}^{t} h\left(t^{\prime}\right) a\left(t-t^{\prime}\right) d t^{\prime}
$$

et $u(t)$ est l'échelon de Heaviside $(u(t)=0$ si $t<0,=1$ si $t>0)$. La représentation (4) est assimilable à une description externe où $h_{0}$ est la réponse impulsionnelle à la condition initiale dans $\Omega$, $h$, est la réponse impulsionnelle à la sollicitation de Dirichlet $p_{1}$ sur $\Gamma_{1}$ et $h_{2}$ est la réponse impulsionnelle à la sollicitation de Neumann $q$ sur $\Gamma_{2}$

\section{Représentation externe par un modèle retard exponentiel}

L'approximation la plus simple des réponses impulsionnelles (5) est donnée par l'exponentielle :

$$
R(\alpha, \eta, t)=\frac{\alpha}{\eta} \mathrm{e}^{-\frac{t}{\eta}} u(t)
$$

La solution $p(r, t)$ sera alors approchée par :

$$
P(r, t)=p_{0}(r)+\sum_{j=0}^{2} R\left(\alpha_{j}(r), \eta_{j}(r), t\right) * a_{j}(t)
$$

où $\left(a_{0}, a_{1}, a_{2}\right)=\left(u, p_{1}, q\right)$. L'intérêt de l'approximation (8) est que l'on a localisé la description. Toute l'information relative à la structure spatiale de la solution est portée par les champs $(\alpha(r), \eta(r))_{r \in \Omega^{\prime}}$ Si l'on connait ces derniers, on peut reconstituer l'évolution temporelle du champ $(P(r, t))_{r \in f}$. Pour reconstituer l'évolution temporelle de $P\left(r^{*}, t\right)$ en un seul point $r^{*}$ fixé (celui de l'instrument), il suffit de connaître $\left(\alpha\left(r^{*}\right), \eta\left(r^{*}\right)\right)$. La connaissance de $P(r, t)$ en d'autres points du domaine n'est pas nécessaire pour reconstituer $P\left(r^{*}, t\right)$, contrairement à une méthode locale de type volumes finis ou éléments finis. C'est cette propriété qui rend l'approche externe bien adaptée à un problème de nature inverse : il n'est pas nécessaire d'expliciter la géométrie d'un problème aux limites. En contrepartie, les paramètres sont susceptibles d'avoir un sens mécanique moins direct.

La réponse indicielle (réponse à un échelon de sollicitation) est donnée par:

$$
R(\alpha, \eta, t) * u(t)=\alpha\left(1-e^{-\frac{l}{\eta}}\right)
$$

Le coefficient $\alpha_{i}(r)$ est égal à la valeur de $p(r, t)$ en régime permanent si la seule sollicitation non nulle a est un échelon. Le coefficient $\eta(r)$ est un temps caractéristique de diffusion. Son rôlé est explicité par l'analyse harmonique:

$$
\begin{aligned}
& R(\alpha, \eta, t)+\sin (\omega t)=g \sin (\omega t+\varnothing), \\
& g=\frac{\alpha}{\sqrt{1+(\omega \eta)^{2}}}, \tan \varnothing=\omega \eta
\end{aligned}
$$

qui fait apparaitre le coefficient d'amortissement $g$ et le déphasage $ø$ pour une sollicitation harmonique de pulsation $\omega$. Pour des sollicitations variant lentement $(\omega \eta$ $<<1$ ), on obtient $g \approx \alpha$ et $\sigma \approx \omega \eta$. Le temps caractéristique $\eta$ quantifie alors le retard entre la sollicitation et la réponse, et $\alpha$ caractérise l'amortissement.

La transitoire (5.a) due aux conditions initiales est approchée, avec le modèle (7), par:

$$
p_{0}(r)+R_{0}\left(\alpha_{0}(r), \eta_{0}(r), t\right) * u(t)=p_{0}(r) e^{-\frac{t}{\eta_{0}(r)}}
$$

Les variations imputables à la sollicitation a $(t)$ (de Dirichlet ou de Neumann) et approchées avec le modèle (7) sont :

$$
R_{j}\left(\alpha_{j}(r), \eta_{j}(r), t\right) * a j(t)=\alpha_{j}(r) A\left(a_{j}, \eta_{j}(r), 0, t\right)
$$

où

$$
A\left(a, \eta, t_{0}, t\right)=\frac{1}{\eta} \int_{t_{n}}^{t} a\left(t^{\prime}\right) e^{-\frac{t-t^{\prime}}{\eta}} d t^{\prime}
$$

est solution de l'équation différentielle $\partial A / \partial t=(a-A) / \eta$.

\section{3}

\section{Modèles d'analyse}

L'instant initial $t=0$ est assimilé à une date pertinente comprise entre la fin de construction et le début du premier remplissage. La grandeur mesurée est supposée influencée : 1) par l'état initial, avec un temps caractéristique $\eta_{0} ; 2$ ) par le niveau de la retenue $\Delta Z(t)=$ $Z(t)-Z_{\text {min' }}$ avec un coefficient d'influence $\alpha_{z}$ et un temps 
caractéristique $\eta_{7} ; 3$ ) par la pluviométrie $Q(t)$, avec un coefficient d'influence $\alpha_{0}$ et un temps caractéristique $\eta_{0}$. Dans ce cas, en un point $r$ fixé (et omis pour simplifier), la forme générale d'un modèle exponential-IRF est:

$$
P(t)=p_{0} e^{-\frac{t}{n_{2}}}+\alpha_{2} A\left(\Delta Z, \eta_{Z}, 0, t\right)+\alpha_{Q} A\left(Q, \eta_{Q}, 0, t\right)+T(t)
$$

La variable explicative $T(t)$ représente les autres effets non stationnaires, dont la formulation sort du cadre des présents développements. On en déduit deux modèles. Le premier est adapté à l'analyse des premières années de vie du barrage pour lequel on suppose que $T(t)$ est négligeable :

$$
P(t)=p_{0} \mathrm{e}^{-\frac{t}{\eta_{t}}}+\alpha_{2} A\left(\Delta Z, \eta_{2}, 0, t\right)+\alpha_{Q} A\left(Q, \eta_{Q}, 0, t\right)
$$

11 apparait de manière naturelle un effet non stationnaire : la dissipation des pressions interstitielles de construction $p_{0}$. Cet effet peut être qualifié d'irréversible. Le second modèle est adapté à l'analyse en exploitation du barrage, à partir d'une date $t_{0 r}$ suffisamment longtemps après sa première mise en eau $\left(t_{0} \gg \eta_{0}\right)$ :

$$
P(t)=C+\alpha_{2} A\left(\Delta Z, \eta_{2^{\prime}}, t\right)+\alpha_{\alpha} A\left(Q, \eta_{(\zeta} 0, t\right)+T(t) \quad(16)
$$

oủ $C$ est une constante égale à la valeur qui serait mesurée au début de la période d'analyse si les sollicitations avaient été nulles avant $t_{0}$. Les cinq paramètres du modèle sont $\left(C, \alpha_{z}, \alpha_{0}\right.$ (linéaires), $\eta_{z}, \eta_{Q}$ (non linéaires)). Puisque :

$$
A(a, \eta, 0, t)=e^{-\frac{t-t_{0}}{\eta}} A\left(a, \eta, 0, t_{0}\right)+A\left(a, \eta, t_{0}, t\right)
$$

on en déduit que :

$$
C=p_{0}-\alpha_{2} \beta_{z}-\alpha_{Q} \beta_{Q}
$$

avec

$$
\beta_{2}=A\left(\Delta Z, \eta_{Z}, 0, t_{0}\right), \beta_{Q}=A\left(Q, \eta_{Q} 0, t_{0}\right) .
$$

Pour une analyse en exploitation, il semble donc indispensable de connaitre les sollicitations antérieures à $t_{0}$ puisque le produit de convolution intègre les variations depuis l'instant initial. Toutefois, à l'aide de (18) et (19), on peut réécrire (16) sous la forme :

$$
P(t)=I(t)+\alpha_{2} A\left(\Delta Z, \eta_{\psi}, t_{\sigma} t\right)+\alpha_{Q} A\left(Q, \eta_{Q^{\prime}} t_{0} t\right)+T(t)
$$
avec

$$
I(t)=p_{0}-\alpha_{2} \beta_{2}\left(1-\mathrm{e}^{-\frac{t-t_{0}}{\eta_{2}}}\right)-\alpha_{Q} \beta_{Q}\left(1-\mathrm{e}^{\frac{t-1 p_{0}}{\eta_{0}}}\right)
$$

Cette fois les paramètres sont $\left(\beta_{2}, \beta_{\alpha} \alpha_{2}, \alpha_{0}\right.$ (linéaires), $\eta_{7}, \eta_{0}$ (non linéaires)). Il est donc possible de s'affranchir de la connaissance des sollicitations antérieures à la période d'analyse en considérant l'influence de ce passé récent comme une inconnue à travers $\left(\beta_{,}, \beta_{0}\right)$. En contrepartie, le modèle a un paramètre supplémentaire, linéaire. Le choix de l'intervalle d'analyse et de l'intervalle de calcul du produit de convolution fait apparaître de manière naturelle l'effet non stationnaire $I(t)$. Cet effet peut être qualifié d'irréversible, mais il n'est en aucun cas assimilable à une dérive ou à un vieillissement.

Le raisonnement général peut être repris pour inclure d'autres effets. Par exemple, dans le cas d'un niveau aval variable $\Delta V(t)=V(t)-Z_{\text {min }}$ qui est une sollicitation de Dirichlet supplémentaire sur une partie de la frontière, le modèle (16) devient:

$$
\begin{aligned}
P(t)=C+ & \alpha_{2} A\left(\Delta Z, \eta_{Z^{\prime}}, 0, t\right)+\alpha_{V} A\left(\Delta V, \eta_{V}, 0, t\right) \\
& +\alpha_{Q} A\left(Q, \eta_{Q} 0, t\right)+T(t)
\end{aligned}
$$

\section{Discrétisation temporelle}

Les modèles ARMA (Auto Regressive Moving Average) sont bien adaptés à l'étude des séries chronologiques. Ils sont utilisés dans de nombreux domaines (Young, 1998), mais encore peu appliqués aux problèmes du génie civil (Owen et al., 2001). Un modèle $\operatorname{ARMA}(p, q)$ consiste à décrire la mesure comme fonction linéaire de $p$ valeurs passées de la réponse (moyenne autorégressive) et de q valeurs passées de la sollicitation (moyenne mobile). Nous allons montrer que le modèle exponential-IRF peut conduire à un modèle ARMA. Le retard exponentiel (7) permet d'écrire la récurrence :

$$
A\left(a, \eta, t_{0}, t+\Delta t\right)=\mathrm{e}^{-\frac{\Delta t}{n}} A\left(a, \eta, t_{0}, t\right)+A(a, \eta, t, t+\Delta t)
$$

En intégrant deux fois par partie (13), il vient :

$$
\begin{gathered}
A(a, \eta, t, t+\Delta t)=a(t+\Delta t)-\eta \frac{\partial a}{\partial t}(t+\Delta t) \\
-e^{-\frac{\Delta t}{\eta}}\left(a(t)-\eta \frac{\partial a}{\partial t}(t)\right)+\eta \int_{0}^{t} \frac{\partial^{2} a}{\partial t^{2}}\left(t^{\prime}\right) e^{-\frac{t+\Delta t-t^{\prime}}{\eta}} d t^{*}
\end{gathered}
$$

Pour une description de la sollicitation en échelons a $(t)$ $=a^{n+1}$ si $t^{n} \leq t<t^{n}+\Delta t^{n}$, on obtient, en notant $A^{n}=$ $A\left(a, \eta, t^{0}, t^{n}\right)$, un modèle ARMA $(1,1)$ :

$$
A^{n+1}=\left(1-\theta_{1}\right) A^{n}+\theta_{1} a^{n+1}, \theta_{1}=1-e^{-\frac{\Delta t^{n}}{n}}
$$

Pour une description en rampes $a(t)=a^{n}+\left(t-t^{n}\right)$ $\left(a^{n+1}-a^{n}\right) / \Delta t^{n}$ si $t^{n} \leq t \leq t^{n}+\Delta t^{n}$, on obtient un modèle ARMA $(1,2)$ :

$$
\begin{gathered}
A^{n+1}=\left(1-\theta_{1}\right) A^{n}+\theta_{1} \theta_{2} a^{n+1}+\theta_{1}\left(1-\theta_{2}\right) a^{n} \\
\theta_{1}=1-\mathrm{e}^{-\frac{\Delta t^{n}}{\eta}}, \theta_{2}=\frac{1}{1-\mathrm{e}^{\frac{\Delta t^{n}}{\eta}}}-\frac{\eta}{\Delta t^{n}}
\end{gathered}
$$

On remarque que $0<\theta_{1}<1$ et $1 / 2<\theta_{2}<1$. Par ailleurs, $\theta_{1} \rightarrow 1$ lorsque $\eta \rightarrow 0$ : ce modèle intégre le cas particulier très important d'une réponse instantanée. On peut obtenir d'autres modèles ARMA(1,q) d'ordre $q$ plus élevé en élevant le degré d'interpolation de la sollicitation. Toutefois, le nombre de paramètres d'ajustement reste constant, contrairement au cas d'une simple application d'un modèle $\operatorname{ARMA}(p, q)$ qui comporte $p+$ $q$ paramètres. Dans les applications, la description de la pluviométrie sera en échelons (25), et la description de l'évolution du niveau de la retenue sera en rampes (26).

\section{5}

\section{Identification du modèle}

On peut obtenir une identification directe en explicitant $\left(\alpha, \eta_{j}\right)$ afin que les deux premiers moments temporels de $h_{j}$ et de $R$ soient égaux pour tout $r$. Cette identification est analytique et suppose que la fonction de Green soit connue. Le moment temporel d'ordre $k$ d'un champ a(r,t) est défini par:

$$
M_{k}[a](r)=\int_{0}^{\infty} t^{k} a(r, t) d t=(-1)^{k} \lim _{s \rightarrow 0} \frac{\partial^{k} \hat{a}}{\partial s^{k}}(r, s)
$$

oủ â(r,s) est la transformée de Laplace de a(r,t). On obtient dans le cas présent: 


$$
\alpha_{j}(r)=M_{0}\left[h_{j}\right](r), \eta_{j}=\frac{M_{1}\left[h_{j}\right](r)}{M_{0}\left[h_{j}\right](r)}
$$

Cette identification sera utilisée pour donner un sens mécanique aux paramètres.

Pour ajuster le modèle sur des mesures in situ, de nombreuses méthodes d'identification sont a priori possibles. Toutefois, le contrôle des sollicitations est impossible; on ne peut pas accéder directement à la réponse impulsionnelle ou a la fonction de transfert. D'autre part, deux raisons conduisent à ne pas réaliser l'identification dans le domaine fréquenciel : 1) les mesures sont susceptibles de comporter des périodes d'interruption (cette situation est souvent rencontrée pour les mesures d'auscultation de barrages) ; 2) le modèle peut être appliqué sur de longues périodes de temps, comprenant des évolutions non périodiques et non stationnaires. Nous sommes donc conduits à réaliser l'identification dans le domaine temporel. Elle est numérique et consiste à calculer les inconnues $(\alpha(r), \eta(r))$ qui minimisent l'écart entre la mesure $Y(r, t)$ et $P(r, t)$ en un point $r$ fixé, et sur un intervalle de temps bien choisi. Le choix de cet intervalie de temps peut être motivé par différentes raisons : élimination des transitoires dues à la condition initiale, analyse d'une période particulière de l'ouvrage, identification du modèle sur une période récente, afin de permettre une prévision des mesures (en général sur les deux ans à venir). Si l'écart est mesuré par une norme quadratique, cette méthode conduit à un problème d'identification par moindres carrés, non linéaire sur les paramètres $(\eta(r))$.

\section{Analyse sur solution exacte}

\section{1}

\section{Problème bidimensionnel}

Afin d'analyser la pertinence du modèle exponential-IRF, on considère le problème bidimensionnel suivant sur domaine rectangulaire $\left.10 \leq x \leq L_{x}, 0 \leq y \leq L_{y}\right)$ homogène anisotrope, pour lequel le repère $(0, x, y)$ coincide avec les axes principaux d'anisotropie :

$$
\begin{gathered}
c \frac{\partial p}{\partial t}(x, y, t)-k_{x} \frac{\partial^{2} p}{\partial x^{2}}(x, y, t)-k_{y} \frac{\partial^{2} p}{\partial y^{2}}(x, y, t)=0 \\
0<x<L_{x}, 0<y<L_{y} \\
p(x, y, 0)=4 p_{\max } \frac{x}{L_{x}}\left(1-\frac{x}{L_{x}}\right)
\end{gathered}
$$

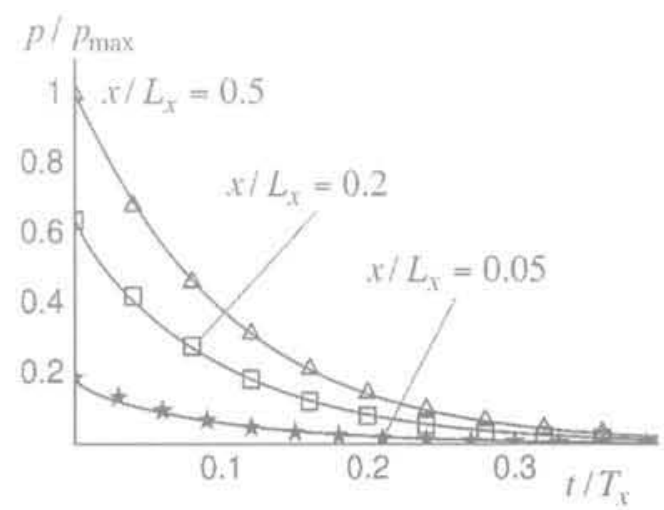

$$
\begin{gathered}
p(0, y, t)=p_{1}(t), p\left(L_{x}, y, t\right)=0, \\
-k_{y} \frac{\partial p}{\partial y}\left(x, L_{y}, t\right)=q(t), \frac{\partial p}{\partial y}(x, 0, t)=0
\end{gathered}
$$

où c est la capacité, $(L, L$,$) sont les dimensions du domaine$ étudié et $\left(k_{x}, k\right)$ sont les conductivités suivant $(0, x)$ et $(0, y)$. Ce problème peut être assimilable, sur une géométrie simplifiée, à un barrage homogène en terre ou au noyau d'un barrage zoné, soumis aux variations de la retenue $p_{f}(t)$ sur sa face amont, au drainage sur sa face aval, à la pluie sur sa crête, et reposant sur une fondation imperméable. La condition initiale peut être assimilable à un champ de pression interstitielle résultant de la construction par couches.

Une représentation de la fonction de Green de ce problème peut être construite à partir de résultats fondamentaux classiques (Morse, 1953 ; Beck et al., 1992 ; Melnikov, 2000) :

$$
\begin{aligned}
& g\left(x, x^{\prime}, y, y^{\prime}, t\right)=\frac{1}{L_{x} L_{y}} g_{3}\left(\frac{x}{L_{x}}, \frac{x^{\prime}}{L_{x}}, \frac{t}{T_{x}}\right) g_{y}\left(\frac{y}{L_{y}}, \frac{y^{\prime}}{L_{y}}, \frac{t}{T_{y}}\right) u(t) \\
& g_{x}\left(r, r^{\prime}, \tau\right)=\frac{1}{2}\left(\theta_{3}\left(\frac{\pi\left(r-r^{\prime}\right)}{2}, \mathrm{e}^{-\pi^{2} t}\right)-\theta_{3}\left(\frac{\pi\left(r+r^{\prime}\right)}{2}, \mathrm{e}^{-\pi^{2} t}\right)\right)(30 \\
& g_{y}\left(r, r^{\prime}, \tau\right)=\frac{1}{2}\left(\theta_{3}\left(\frac{\pi\left(r-r^{\prime}\right)}{2}, \mathrm{e}^{-\pi^{2} t}\right)+\theta_{3}\left(\frac{\pi\left(r+r^{\prime}\right)}{2}, \mathrm{e}^{-\pi^{2} t}\right)\right)
\end{aligned}
$$

où $\left(T_{x}, T_{v}\right)$ sont des temps caractéristiques définis par

$$
T_{x}=\frac{c L_{x}^{2}}{k_{x}}, T_{y}=\frac{c L_{y}^{2}}{k_{y}}
$$

et où $\theta_{3}$ est la fonction elliptique de troisième espèce

$$
\begin{aligned}
\theta_{3}\left(\frac{\pi \xi}{2}, e^{-\pi^{2} \tau}\right) & =\frac{1}{\sqrt{\pi \tau}} \sum_{k=-\infty}^{+\infty} \exp \left(-\frac{(\xi-2 k)^{2}}{4 \tau}\right) \\
& =1+2 \sum_{k=1}^{+\infty} \cos (k \pi \xi) \mathrm{e}^{-(k \pi)^{2} \tau}
\end{aligned}
$$

\section{2}

\section{Transitoire initiale}

A l'aide de (5a), (28) et (30), l'identification directe conduit aux résultats exacts suivants :

$$
\alpha_{0}(x, y)=-p_{0}(x, y), \eta_{0}(x, y)=\frac{1}{12}\left(-\left(\frac{x}{L_{x}}\right)^{2}+\frac{x}{L_{x}}+1\right) T_{x}
$$

La transitoire exacte représentant la dissipation des pressions initiales est approchée avec une bonne précision par (11) et (33) (Fig. 2).

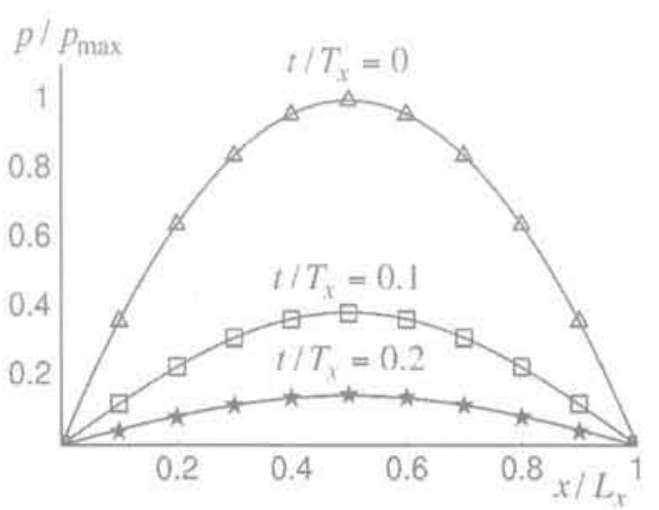

FG, 2 Condition initiale, évolution temporelle et répartition spatiale (traits pleins: solution exacte, symboles : modèle retard).

Initial condition, temporal evolution and spatial profile (solid lines : exact solution, symbols : delayed response model), 


\section{Sollicitation de Dirichlet}

A l'aide de (5b), (28) et (30), l'identification directe conduit aux résultats exacts suivants :

$$
\alpha_{1}(x, y)=1-\frac{x}{L_{x}}, \eta_{1}(x, y)=\frac{x}{6 L_{x}}\left(2-\frac{x}{L_{x}}\right) T_{x}
$$

La réponse indicielle, représentant l'augmentation de pression provoquée par un échelon de retenue, est approchée avec une précision convenable par (9) et (34) (Fig. 3). La figure 4 montre la réponse à une sollicita- tion harmonique (10) pour $\omega T=2 \pi$. Trois aspects essentiels sont reproduits : 1) l'hystérésis d'un cycle de sollicitation (Fig. 4a) ; 2) pour une même valeur de la sollicitation, la réponse a une valeur différente suivant son évolution (Fig. 4b) ; 3) la réponse peut avoir une valeur supérieure à la sollicitation (Fig. 4b).

Le domaine de validité du modèle exponential-IRF (7) peut être estimé par les diagrammes logarithmiques (Fig. 5). Le gain et la phase sont correctement reproduits par les approximations (10) pour $\omega T \leq 10$ environ. La figure 5 met en évidence les limites du modèle : il ne peut pas rendre compte des harmoniques élevées des sollicitations.
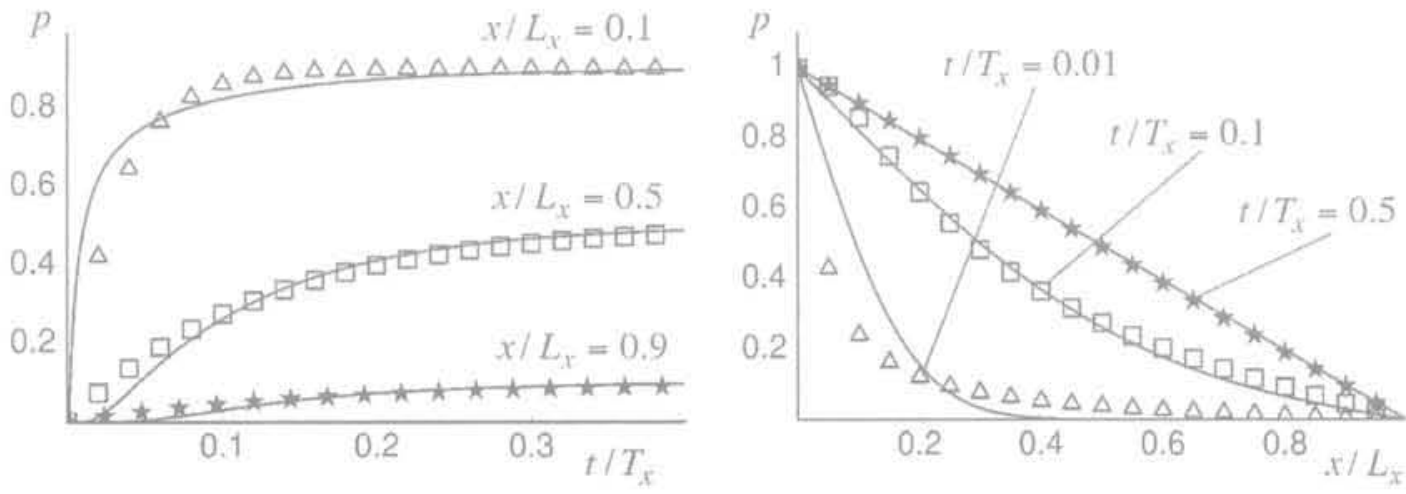

FG. 3 Échelon de Dirichlet, évolution temporelle et répartition spatiale (traits pleins: solution exacte, symboles: modèles retard).

Dirichlet unit step, temporal evolution and spatial profile (solid lines : exact solution, symbols : delayed response model).
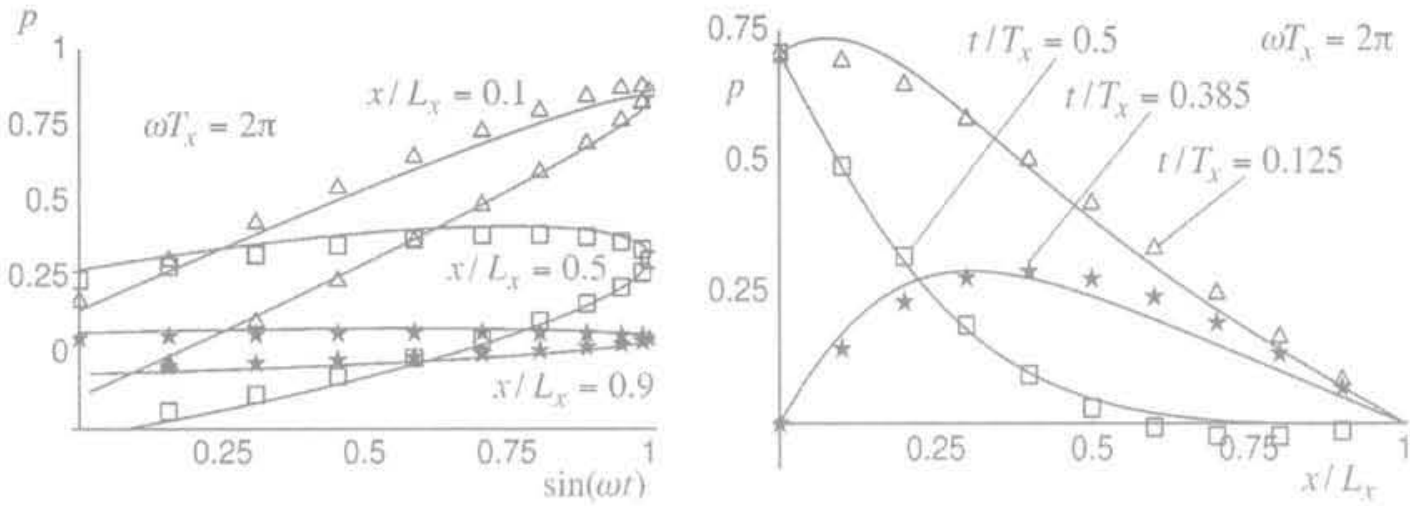

FIG.4 Sollicitation harmonique, évolution temporelle et répartition spatiale (traits pleins: solution exacte, symboles : modèles retard).

Harmonic loading, temporal evolution and spatial profile (solid lines : exact solution, symbols : delayed response model).
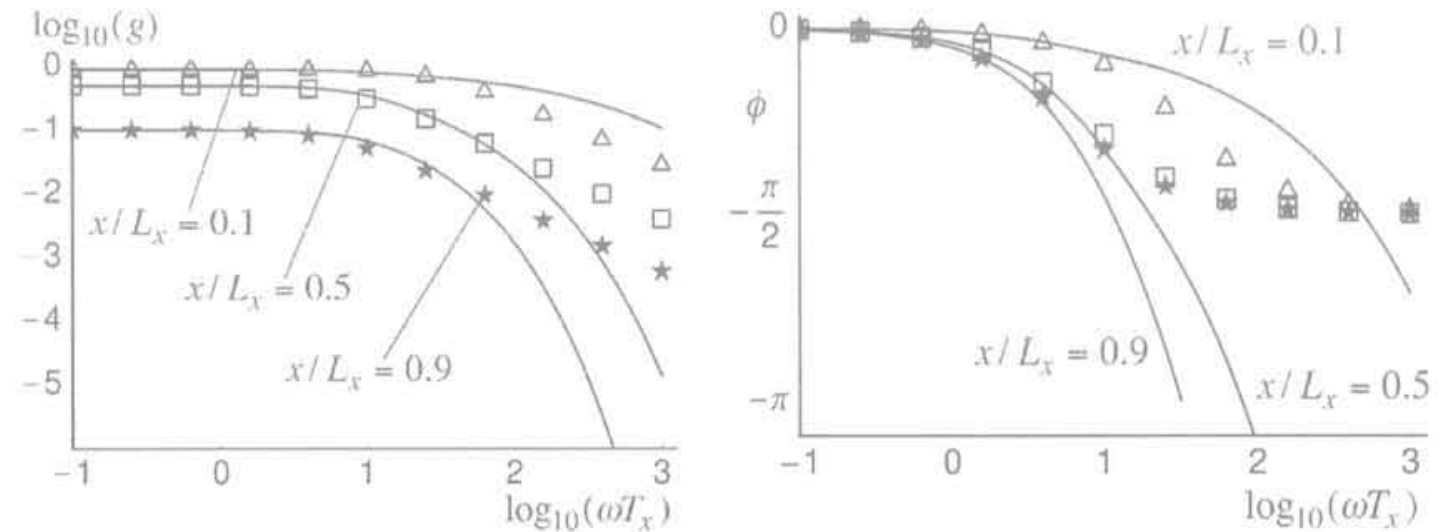

FG. 5 Sollicitation harmonique, diagrammes logarithmiques (traits pleins: solution exacte, symboles: modèles retard), Harmonic loading, logarithmic diagrams (solid lines: exact solution, symbols: delayed response model). 


\section{Sollicitation de Neumann}

A l'aide de (5c), (28) et (30), l'identification directe conduit à des expressions complexes sous forme de séries infinies. On peut déduire les résultats approchés suivants pour $T_{y} \geq T_{x}$ :

$$
\begin{gathered}
\alpha_{2}(x, y) \approx \frac{4 \sin \left(\pi \frac{x}{L_{x}}\right) \cosh \left(\pi a \frac{y}{L_{y}}\right)}{a \pi^{2} \sinh (\pi a)} \frac{L_{y}}{K_{y}} \\
\eta_{2}(x, y) \approx \frac{1}{2 \pi^{2} a}\left(1+\pi a-\pi a \frac{y}{L_{y}} \tanh \left(\pi a \frac{y}{L_{y}}\right)\right) \sqrt{T_{x} T_{y}}
\end{gathered}
$$

où $\mathrm{a}=\sqrt{T_{y} / T_{x}}$ rend compte de l'influence de l'anisotropie et des dimensions caractéristiques. Le coefficient $\alpha_{2}(x, y)$ a la dimension d'un temps. Le temps caractéristique $\eta_{2}$ peut être considéré comme indépendant de $x$, mais il intègre l'influence de $a$, de nature bidimensionnelle. L'approximation (9) et (35), représentant l'augmentation de pression provoquée par un échelon de pluie, reproduit correctement la solution exacte en temps et en espace, dans les deux dimensions spatiales (Figs, 6, 7, 8 et 9). Pour un échelon de pluie, la vitesse d'évolution de la pression augmente avec a (Figs. 6a, 7a, 8a et 9a). La profondeur d'influence diminue avec a (Figs. $6 \mathrm{~b}$ et $7 \mathrm{~b}$ ), de même que la valeur maximale de la pression (Fig, 8 b et 9b)
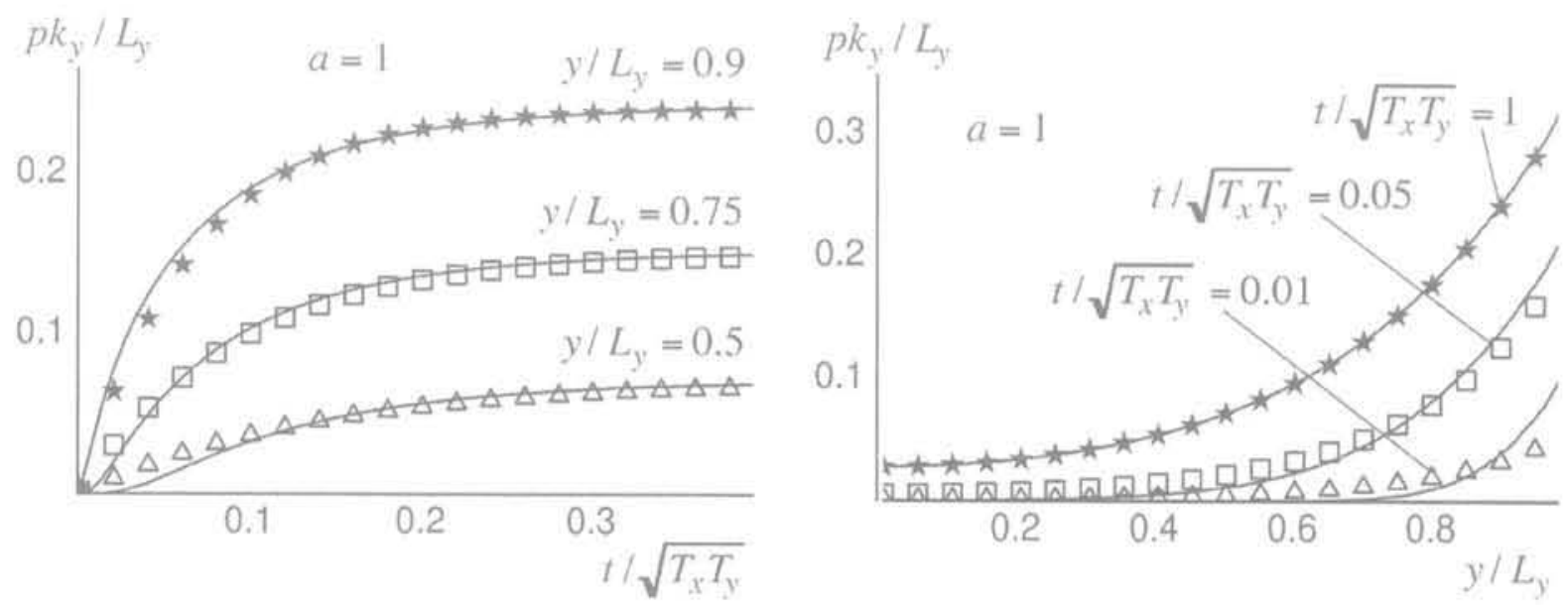

FIG.6 Échelon de Neumann, évolution temporelle et répartition spatiale pour $x / L_{x}=0,3$ et $a=1$ (traits pleins: solution exacte, symboles: modèles retard).

Neumann unit step, temporal evolution and spatial profile for $x / L_{x}=0.3$ and $a=1$ (solid lines: exact solution, symbols: delayed response model).
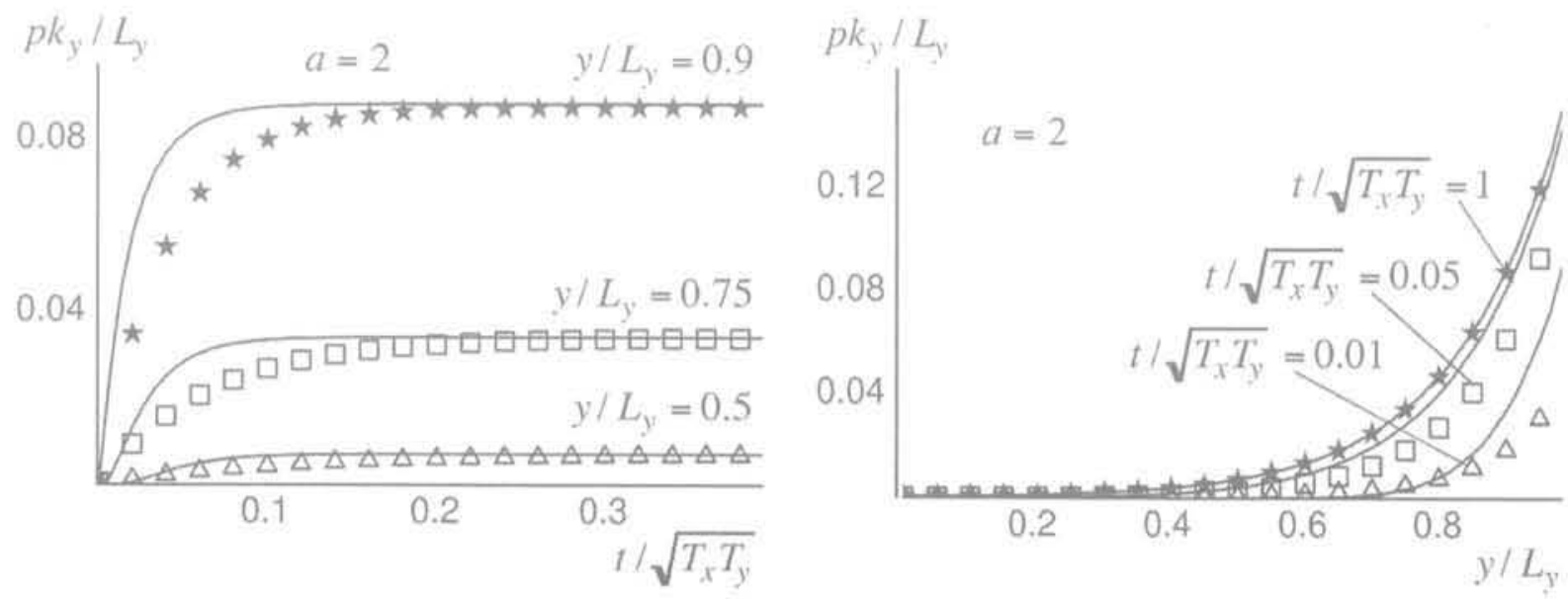

FG.7 Échelon de Neumann, évolution temporelle et répartition spatiale pour $x / L_{x}=0,3$ et $a=2$ (traits pleins: solution exacte, symboles: modèles retard).

Neumann unit step, temporal evolution and spatial profile for $x / L_{x}=0.3$ and $a=2$ (solid lines: exact solution, symbols: delayed response model). 

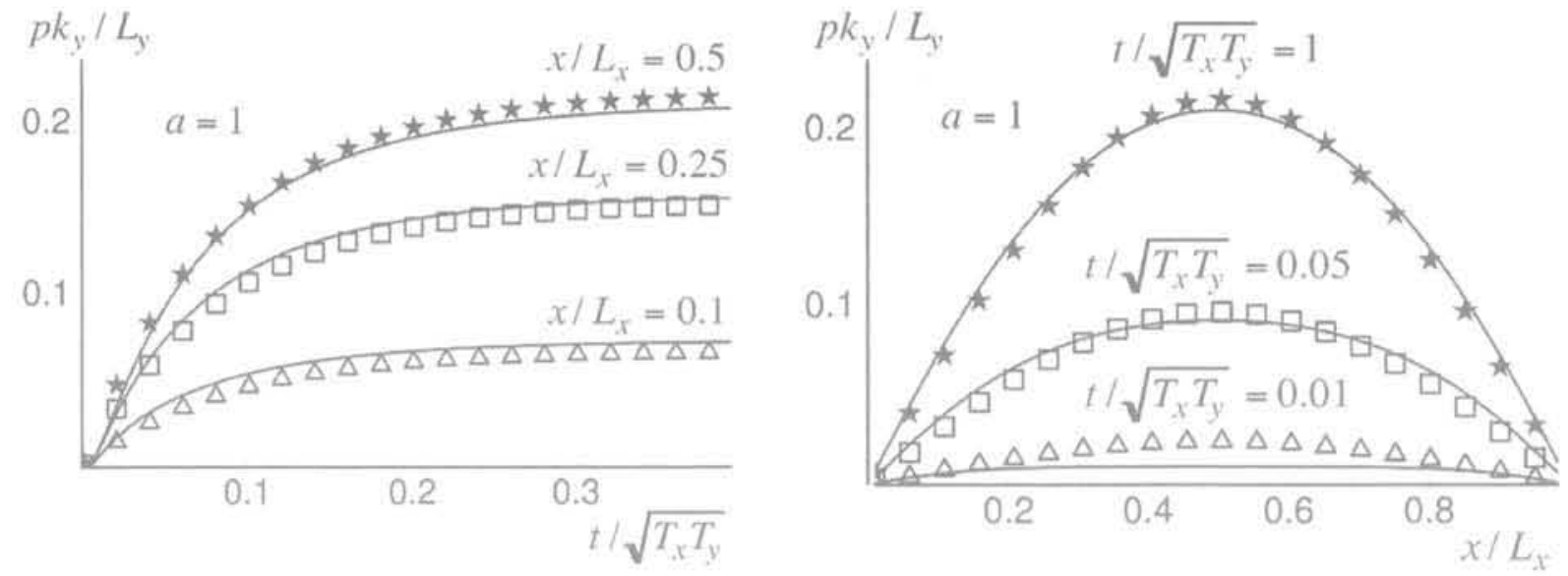

FG. 8 Échelon de Neumann, évolution temporelle et répartition spatiale pour $y / L_{y}=0,8$ et a $=1$ (traits pleins: solution exacte, symboles: modèles retard).

Neumann unit step, temporal evolution and spatial profile for $y / L_{2}=0.8$ and $a=1$ (solid lines: exact solution, symbols: delayed response model).
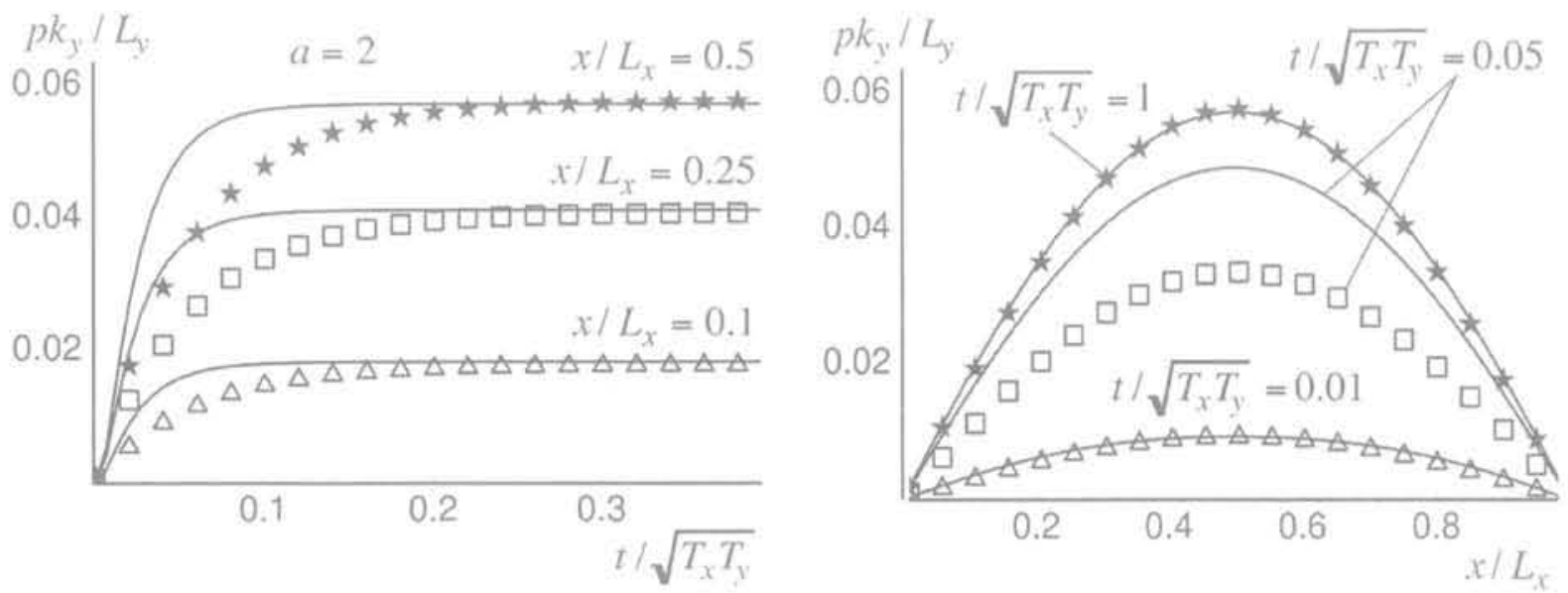

FG:9 Échelon de Neumann, évolution temporelle et répartition spatiale pour $y \Lambda_{y}=0,8$ et $a=2$ (traits pleins : solution exacte, symboles : modèles retard).

Neumann unit step, temporal evolution and spatial profile for $y / h_{1}=0.8$ and $a=2$ (solic lines: exact solution, symbols: delayed response model).

\section{5}

\section{Signification mécanique des paramètres}

Ayant noté $\left(\alpha_{1}, \eta_{1}, L_{x}\right)$ les grandeurs relatives à la description simplifiée (29), nous noterons $\left(\alpha_{2}, \eta_{2}, L_{2}\right)$ les paramètres associés à la partie hydrostatique du modèle exponential-IRF, et identifiés sur mesures d'auscultation. Lorsque la description (29) est pertinente, le résultat (34) permet une interprétation mécanique. C'est en particulier le cas d'instruments situés dans le corps d'un barrage en terre ou dans le noyau d'un barrage zoné. La longueur $L_{z}$ peut être assimilée à une distance moyenne de drainage, entre la sollicitation (retenue parement amont), et l'exutoire (drain cheminé, tapis drainant, parement aval) (Fig. 10). Il est possible de vérifier la cohérence des grandeurs $\left(x, L_{7}\right)$ estimées sur plan et du paramètre $\alpha$, identifié sur les mesures.

Le coefficient d'amortissement statique $\alpha_{7}$ contient deux informations : 1) l'efficacité du drainage (drain vertical, tapis drainant, puits de décompression) ou des dispositifs d'étanchéité (voiles d'injection) ; 2) la position de l'instrument sur le chemin de drainage par rapport au parement amont. Ce chemin de drainage peut être supérieur au chemin représenté sur la figure 10 en cas de colmatage du drain, lorsque la nappe atteint le parement aval. Un coefficient $\alpha$, proche de l'unité signifiera que l'instrument est proche du parement amont (x petit) ou que l'exutoire est loin de la sollicitation ( $L_{z}$ grand).

Le temps caractéristique local de l'înfluence de la retenue $\eta_{7}$ contient deux informations : 1) l'efficacité du drainage par l'intermédiaire du carré de $L_{z} ; 2$ ) la diffusivité de la zone instrumentée $D=k / c$, grandeur matérielle qui dépend d'une perméabilité $k$ et de la capacité c. Dans le cas anisotrope, l'interprétation est plus complexe. Toutefois, $\eta$ permet d'accéder à une estimation de cette diffusivité si $L_{z}$ est connue. A l'aide de (31) et (34), on obtient :

$$
D=\frac{\left(1-\alpha_{Z}^{2}\right) L_{Z}^{2}}{6 \eta_{2}}
$$




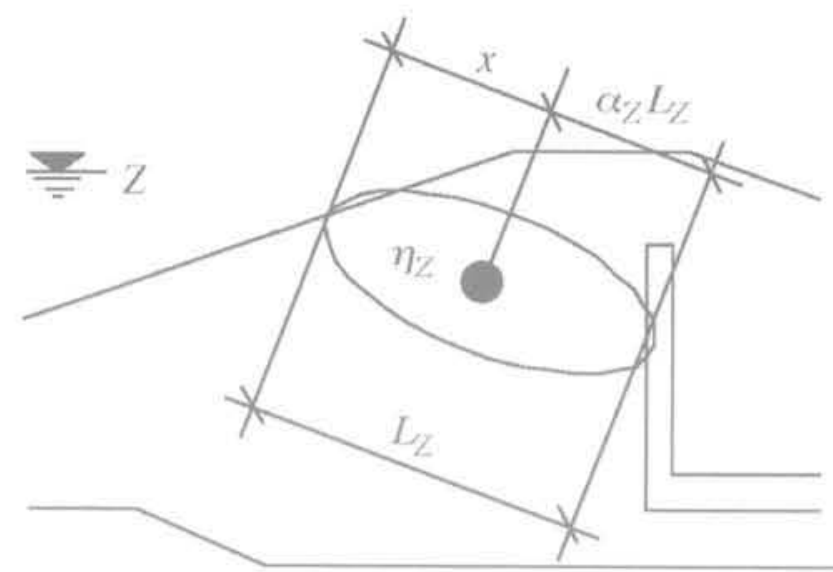

Fig. 10 Les paramètres $\alpha_{2}$ et $\eta_{z}$ peuvent être interprétés pour des mesures dans le corps d'un barrage: $\alpha_{z}$ est la distance relative du point de mesure à l'exutoire, $\eta_{2}$ est le temps caractéristique du point de mesure, $L_{z}$ est la longueur moyenne de drainage.

Parameters $\alpha$, and $\eta$, can be specified for an instrument placed in the body of the dam: $\alpha$, is the relative distance of the measurement point to the drainage system, $\eta_{-}$is the characteristic response time of the measurement point, $L$ is the mean length of the drainage path.

Un temps $\eta$, très grand caractérisera un sol très peu perméable, ou une longueur de drainage $L_{z}$ très importante. Un temps $\eta$, non nul caractérisera une capacité non nulle.

Dans les zones de marnage, le sol est susceptible d'être non saturé, ce qui explique une capacité non nulle. Dans les zones situées sous le niveau de la surface libre, cette capacité peut également être non nulle.

La compressibilité du sol peut être une première raison. Toutefois, les matériaux fins utilisés dans les barrages sont de faible plasticité (indice de plasticité $I_{\text {in }}<$ $35 \%$ ), compactés à la mise en place donc surconsolidés en partie haute. Par ailleurs, ils sont déjaugés sous la surface libre. En phase d'exploitation, dans le corps du barrage ou en aval de l'ouvrage et dans les terrains naturels, le sol travaille sous des sollicitations déjà subies. En première approximation, on peut donc considérer que, dans ce cadre, c'est le module de rigidité élastique tangent qui quantifie l'amplitude des variations de pression interstitielle provoquée par la déformation de la matrice solide. L'influence du couplage hydromécanique sur la capacité est probablement du second ordre.

La compressibilité du fluide interstitiel peut être la raison principale. Saint-Arnaud (1995) propose en particulier de prendre en compte le fait que l'eau dans le corps d'un barrage contienne, en plus de sa teneur en air naturelle issue de la retenue, de l'air emprisonné au moment de la mise en eau, en partie comprimé et en partie dissous. La partie dissoute est transportée à travers le corps du barrage et s'échappe à l'aval.

La conclusion est que la capacité peut ne pas être nulle sous la surface libre. Dans ce cas, les réponses en pression interstitielle à des sollicitations seront retardées. Ces réponses pourront toutefois être considérées comme instantanées pour des temps caractéristiques inférieurs au temps de réponse des appareils d'auscultation ou à la périodicité de mesure.
Nous noterons $\left(\alpha_{0}, \eta_{\rho}\right)$ les paramètres du modèle exponential-IRF relatifs à la pluie, et identifiés sur mesures d'auscultation. Les mesures réalisées dans le corps du barrage étant le plus souvent peu influencées par la pluie, l'interprétation mécanique de ces paramètres ne serait utile que pour des instruments situés en terrain naturel, dans les abords du barrage. Malheureusement, dans ce cas on dispose rarement de relevés ou de plans, et une interprétation ne peut être proposée sur la base du problème (29) et des résultats (35), beaucoup trop simplistes. On peut toutefois remarquer que $\alpha_{0}$ a la dimension d'un temps indépendant de la capacité $c$. On peut assimiler $\alpha_{0}$ à un temps de drainage, nécessaire pour que l'eau de pluie infiltrée arrive à l'exutoire le plus proche dans la zone auscultée par l'instrument. Plus l'eau infiltrée est évacueje rapidement, plus $\alpha_{0}$ est petit et moins l'effet pluie influence le niveau mesuré. On remarque que si la seule connaissance de la diffusivité $D$ suffit à caractériser la réponse à une sollicitation en charge, il est nécessaire de connaitre la diffusivité et la perméabilité pour caractériser la réponse à une sollicitation en flux. Il est alors normal d'obtenir dans ce cas deux temps caractéristiques $\alpha_{0}$ et $\eta_{0}$ ce dernier étant le temps de diffusion relatif au point ausculté et à l'effet pluie.

\section{Applications}

\section{Analyse retard de l'effet retenue}

Les mesures des instruments d'un barrage en terre homogène de 30 mètres de haut, avec tapis drainant horizontal, sont analysées avec le modèle (16) sans effet pluie. Le corps du barrage est en sable argileux (perméabilité comprise entre $10^{-5}$ et $10^{-7} \mathrm{~m} / \mathrm{s}$ ). Le talus aval est ausculté par sept piézomètres suivant trois coupes (Fig. 11a). L'axe du barrage est ausculté par six cellules de pression interstitielle à cordes vibrantes sur trois coupes (Fig. 11b). Trois cellules sont situées en fondation.

Le piézomètre évoqué (Fig. 1) est situé en pied de parement aval (P1 Fig.11a). Il est analysé en deux phases. L'ajustement du modèle exponential-IRF sur la phase de remplissage ( 150 jours) conduit à $\alpha_{z}=0,44$ et $\eta_{7}=83$ jours (Fig. 12). Sur la première phase d'exploitation ( 2280 jours), on obtient $\alpha_{\text {. }}=0,22$ et $\eta_{z}=159$ jours (Fig. 13). L'évolution de ces valeurs entre les deux phases peut s'expliquer par la saturation progressive du sol à la suite du premier remplissage. La figure 14 compare les mesures et le modèle pour la cellule CV13 située dans le corps du barrage (Fig. 11b). Les figures $12 \mathrm{~b}, 13 \mathrm{~b}$ et $14 \mathrm{~b}$ montrent l'hystérésis sur un cycle hausse/diminution du niveau de la retenue, ainsi qu'un décalage de la réponse de l'ordre de grandeur du temps caractéristique $\eta_{2}$

Le tableau I donne les résultats de l'analyse retard des six cellules (Fig. 11b), ainsi qu'une estimation de la diffusivité. Celle-ci est d'un ordre de grandeur supérieur dans les zones situées sous la surface libre $\left(10^{-4}\right.$ à $\left.10^{-3} \mathrm{~m}^{2} / \mathrm{s}\right)$ par rapport aux zones de marnage $\left(10^{-5}\right.$ à $10^{-4} \mathrm{~m}^{2} / \mathrm{s}$ ), susceptibles d'être non saturées et d'avoir une capacité moyenne plus èlevée.

Le tableau II donne les résultats de l'analyse retard 

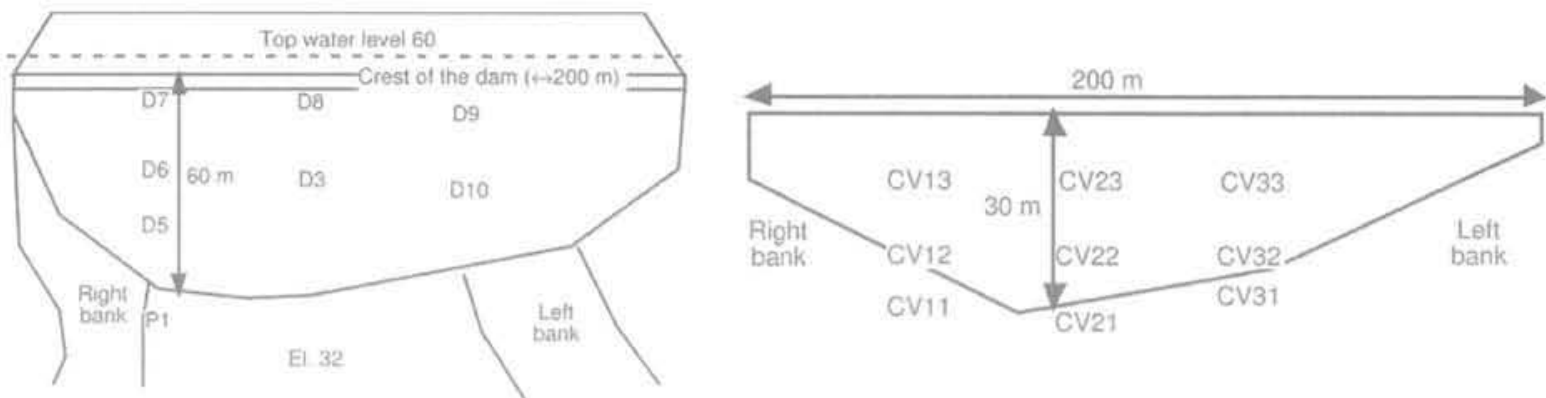

FiG.11 Vue en plan et implantation des piézomètres, profil en long de l'axe (vue de l'aval) et implantation des cellules de pression interstitielle.

Location map of piezometers in the plan of the dam site, location map of the cells in the longitudinal profile (from downstream)

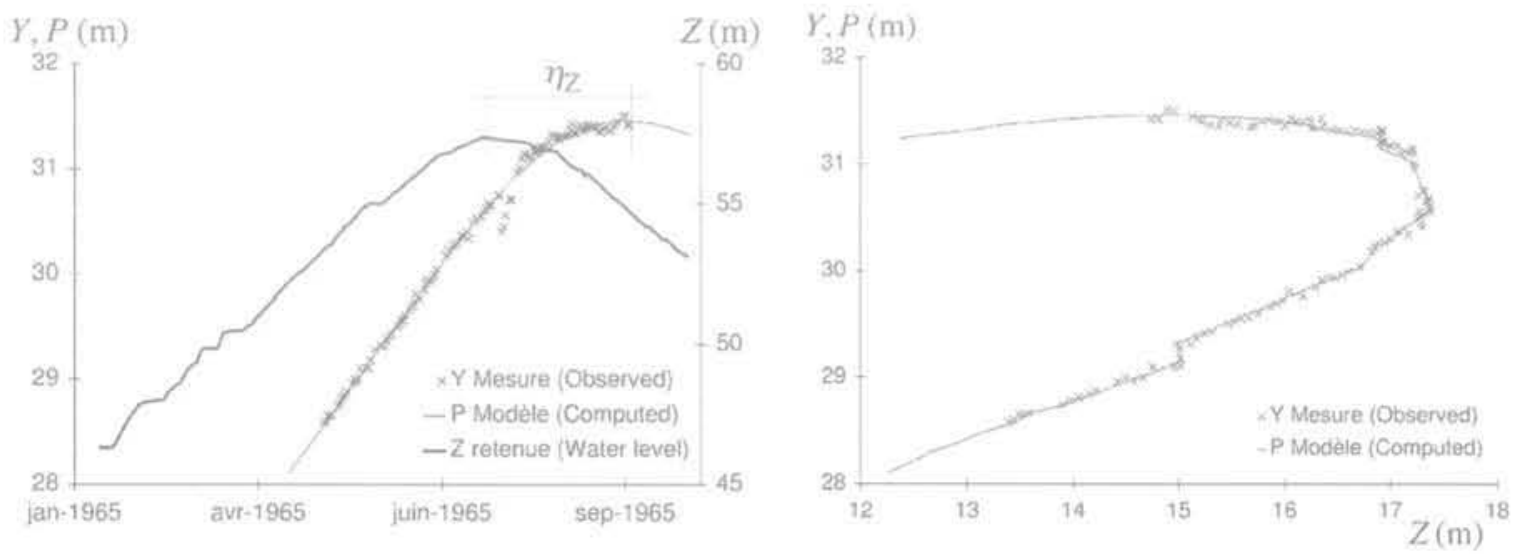

FG. 12 Analyse retard du piézomètre P1 durant le remplissage, niveaux piézométriques en fonction du temps et en fonction de la retenue ( $\eta_{2} \approx 83$ jours).

Delayed response analysis of $\mathrm{P} 1$ piezometer during the impounding phase, piezometric head $v s$, time $\left(\eta_{z} \approx 83\right.$ days) piezometric head vs, water level.
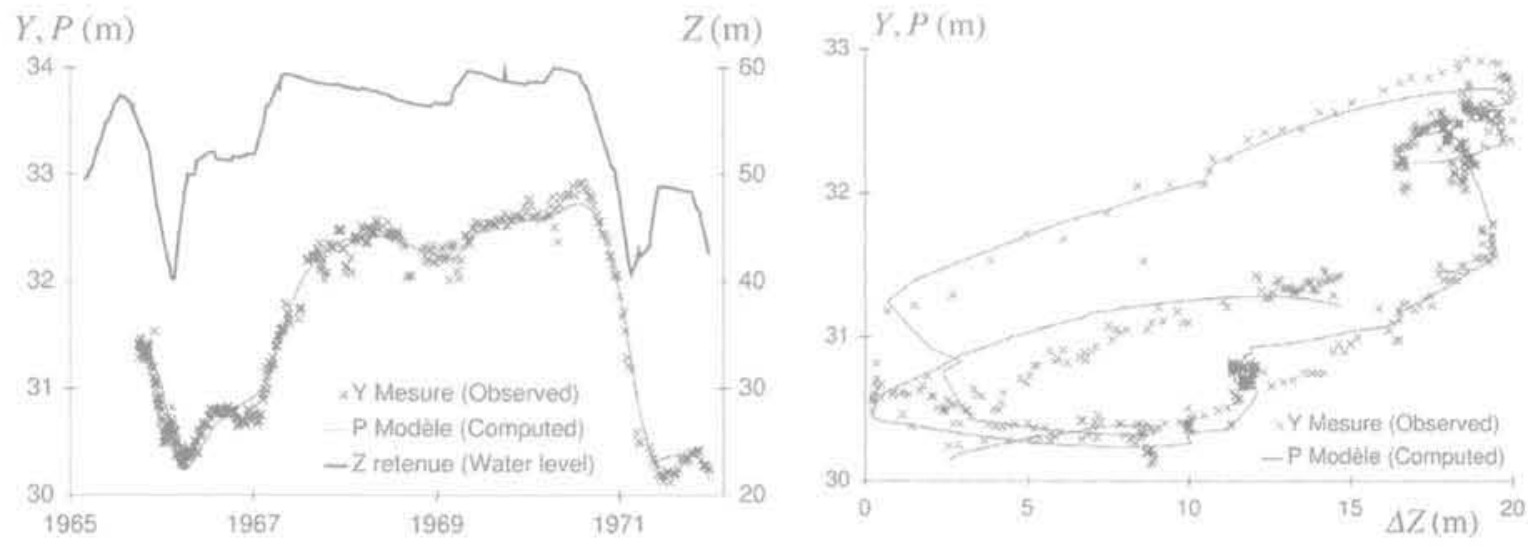

FIG. 13 Analyse retard du piézomètre P1 durant l'exploitation, niveaux piézométriques en fonction du temps et en fonction de la retenue.

Delayed response analysis of P1 piezometer during the exploitation phase, piezometric head vs. time piezometric heac vs. water level.

des piézomètres auscultant le talus aval (Fig. 11a). Ce tableau contient également les cellules auscultant l'axe du barrage, afin de vérifier la cohérence des résultats. Malgré la grande différence de principe entre les deux instruments, les diffusivités estimées sont du même ordre de grandeur. On peut l'expliquer par le fait que l'analyse intègre implicitement les informations relatives à une zone située entre le parement amont et l'instrument, plus étendue que la zone directement concernée par l'instrument. Le tableau II montre que la diffusivité semble plus importante en rive droite, ce qui peut correspondre à une perméabilité plus importante. 

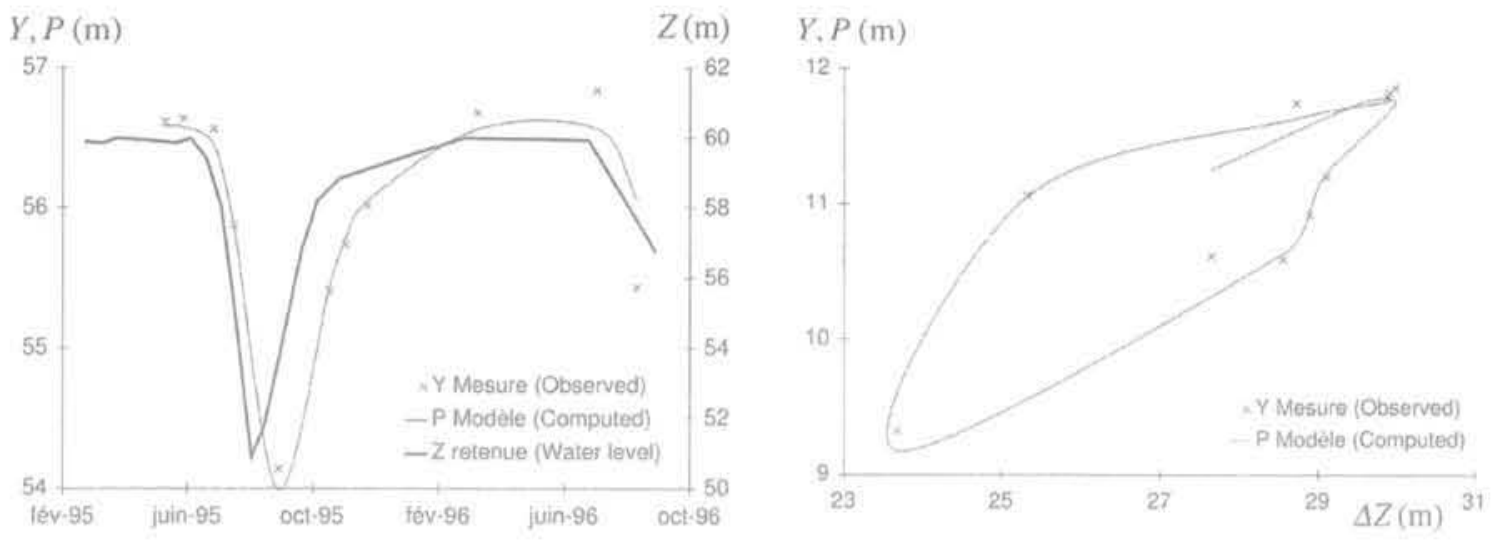

FiG. 14 Analyse retard de la cellules CV13, niveaux piézométriques en fonction du temps et en fonction de la retenue ( $\eta_{z} \approx 23$ jours).

Delayed response analysis of CV13 cell, piezometric head vs, time, piezometric head vs, water level $\left(\eta_{z}=23\right.$ days).

tABLEAU1 Résultats de l'analyse retard des mesures de cellules (Fig. 11b).

Results of delayed response analysis of cells data (Fig. 11b).

\begin{tabular}{|c|c|c|c|}
\hline Cellule & $\alpha_{z}$ & $\begin{array}{c}\eta_{z} \\
\text { (jours) }\end{array}$ & $\begin{array}{l}\text { Diffusivité } \\
\left(10^{-6} \mathrm{~m}^{2} / \mathrm{s}\right)\end{array}$ \\
\hline \multicolumn{4}{|c|}{ Niveau 52 NGF (zone de marnage) } \\
\hline $\begin{array}{l}\text { CV33 } \\
\text { CV23 } \\
\text { CV13 }\end{array}$ & $\begin{array}{l}0,27 \\
0,38 \\
0,39\end{array}$ & $\begin{array}{l}33 \\
33 \\
23\end{array}$ & $\begin{array}{l}4 \\
5 \\
8\end{array}$ \\
\hline
\end{tabular}

Niveau 40 NGF (zone saturée)

\begin{tabular}{|c|c|c|c|}
\hline $\begin{array}{l}\text { CV32 } \\
\text { CV22 } \\
\text { CV12 }\end{array}$ & $\begin{array}{l}0,47 \\
0,49 \\
0,38\end{array}$ & $\begin{array}{l}25 \\
33 \\
10\end{array}$ & $\begin{array}{r}60 \\
50 \\
100\end{array}$ \\
\hline \multicolumn{4}{|l|}{ Fondation } \\
\hline $\begin{array}{l}\text { CV31 } \\
\text { CV21 } \\
\text { CV11 }\end{array}$ & $\begin{array}{l}0,43 \\
0,43 \\
0,51\end{array}$ & $\begin{array}{l}28 \\
56 \\
32\end{array}$ & $\begin{array}{r}100 \\
60 \\
100\end{array}$ \\
\hline
\end{tabular}

\section{2}

\section{Analyse retard de l'effet pluie}

L'analyse de quatre piézomètres de rive d'un barrage zoné situé dans une région où se produisent des précipitations courtes mais violentes permet d'évaluer le modèle "pluie». Deux piézomètres sont situés en rive droite (PZ17 et PZ14) et deux autres en rive gauche (PZ18 et PA4). Le tableau III donne les résultats des analyses avec le modèle (16). L'influence de la retenue est instantanée $\left(\eta_{7} \approx 0\right)$. Les parts explicatives sont calculées à partir des résidus partiels et correspondent au ratio variation de la variable explicative/variation totale. L'influence de la pluie est importante (de 19\% à $52 \%$ des variations mesurées). Une étude théorique complémentaire ainsi qu'une bonne connaissance de l'ouvrage sont nécessaires pour interpréter les temps caractéristiques $\left(\alpha_{Q}, \eta_{Q}\right)$. La reconstitution des variations est
TABLEAUII Résultats de l'analyse retard des mesures de cellules et de piézomètres (Fig. 11). Results of delayed response analysis of cells and piezometers data (Fig. 11).

\begin{tabular}{|c|c|c|c|}
\hline Cellule & $\alpha_{2}$ & $\underset{\text { (jours) }}{\eta_{z}}$ & $\begin{array}{l}\text { Diffusivité } \\
\left(10^{-5} \mathrm{~m}^{2} / \mathrm{s}\right)\end{array}$ \\
\hline \multicolumn{4}{|c|}{ Coupe rive gauche } \\
\hline $\begin{array}{l}\text { CV33 } \\
\text { CV32 } \\
\text { PID9 } \\
\text { PID10 }\end{array}$ & $\begin{array}{l}0,27 \\
0,47 \\
0,42 \\
0,23\end{array}$ & $\begin{array}{l}33 \\
25 \\
45 \\
45\end{array}$ & $\begin{array}{r}4 \\
60 \\
40 \\
40\end{array}$ \\
\hline \multicolumn{4}{|c|}{ Coupe centre } \\
\hline $\begin{array}{l}\text { CV23 } \\
\text { CV22 } \\
\text { PID8 } \\
\text { PID3 }\end{array}$ & $\begin{array}{l}0,38 \\
0,49 \\
0,29 \\
0,21\end{array}$ & $\begin{array}{l}33 \\
33 \\
30 \\
31\end{array}$ & $\begin{array}{r}5 \\
50 \\
30 \\
50\end{array}$ \\
\hline \multicolumn{4}{|c|}{ Coupe rive droite } \\
\hline $\begin{array}{l}\text { CV13 } \\
\text { CV12 } \\
\text { PID7 } \\
\text { PID6 } \\
\text { PID5 }\end{array}$ & $\begin{array}{l}0,39 \\
0,38 \\
0,28 \\
0,53 \\
0,16\end{array}$ & $\begin{array}{l}23 \\
10 \\
18 \\
22 \\
91\end{array}$ & $\begin{array}{r}8 \\
100 \\
50 \\
100 \\
20\end{array}$ \\
\hline
\end{tabular}

convenable et montre les pics provoqués par la pluie (Fig. 15a). Les graphes relatifs à l'effet pluie sont tracés (Fig. 15b), où $P_{0}(t)=\alpha_{0} A\left(Q, \eta_{\gamma} 0, t\right)$ et $Y_{0}(t)=P_{0}(t)+Y(t)-$ $P(t)$. Ce modèle "pluie) apparait comme perfectible car il ne reproduit que quelques pics et quelques creux de piézométrie.

L'utilisation du modèle retard pour l'effet «pluie » représente un progrès, mais les résultats ne sont pas aussi bons que pour l'effet « retenue ». On peut avancer trois raisons à cela : 1) toute la pluie ne s'infiltre pas, les quantités d'eau de pluie infiltrées et ruisselées dépendent de l'importance des précípitations, de la pente et de la perméabilité du terrain ; 2) le chemin d'écoulement de l'eau infiltrée débute à la surface, et passe par une zone non saturée, mal expliquée par un modèle linéaire à diffusivité constante ; 3) la pluviométrie peut comporter des harmoniques élevées, mal prises en compte par le modèle exponentiel $\left(\omega T_{Q}>10\right)$. 

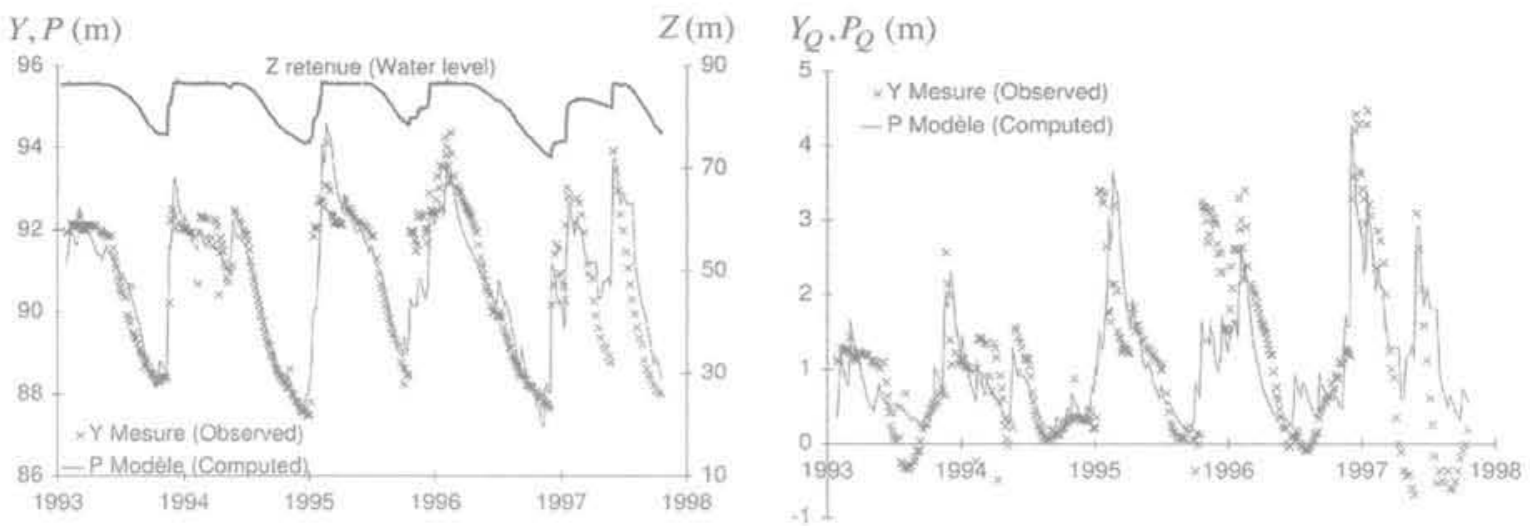

FiG. 15 Analyse retard du piézomètre PZ17, niveaux piézométriques en fonction du temps, influence de la pluie en fonction du temps.

Delayed response analysis of PZ17 piezometer, piezometric head vs, time, rainfall effect vs, time.

TABLEAU III Résultats de l'analyse retard des mesures de piézomètres.

Results of delayed response analysis of piezometer data.

\begin{tabular}{c|c|c|c|c|c|c} 
Piézomètre & $\begin{array}{c}\text { Part } \\
\text { explicative } \\
\text { retenue }\end{array}$ & $\begin{array}{c}\text { Part } \\
\text { explicative } \\
\text { pluie }\end{array}$ & $\alpha_{2}$ & $\begin{array}{c}\eta_{\bar{z}} \\
\text { (jours) }\end{array}$ & $\begin{array}{c}\alpha_{0} \\
\text { (jours) }\end{array}$ & $\begin{array}{c}\eta_{0} \\
\text { (jours) }\end{array}$ \\
\hline PZ17 & $60 \%$ & $24 \%$ & 0,32 & 0 & 273 & 38 \\
PZ14 & $54 \%$ & $23 \%$ & 0,14 & 0 & 815 & 33 \\
PZ18 & $62 \%$ & $19 \%$ & 1,00 & 0 & 147 & 32 \\
PA4 & $21 \%$ & $52 \%$ & 0,46 & 0 & 1016 & 32 \\
\hline
\end{tabular}

\section{Analyse retard des effets de la retenue et de la pluie}

Les données d'auscultation d'un barrage homogène de 15,5 mètres de haut sont analysées avec le modèle (16). La fondation est constituêe d'arènes granitiques (perméabilité comprise entre $10^{-5}$ et $10^{-6} \mathrm{~m} / \mathrm{s}$ ). Elle est étanchée par un voile d'injection. Le remblai comporte des arènes (perméabilité comprise entre $10^{-7}$ et $10^{-8} \mathrm{~m} / \mathrm{s}$ ) et un drain cheminée. Les treize piézomètres à lecture directe auscultent les abords du barrage (Fig, 16a). Les six cellules de pression interstitielle à corde vibrante sont situées dans l'axe de l'évacuateur (Fig. 16b).

Le tableau IV donne les résultats des analyses des six cellules à cordes vibrantes, qui ne sont pas influen-

\begin{tabular}{|c|c|c|c|}
\hline TABLEAU IV & \multicolumn{3}{|c|}{$\begin{array}{l}\text { Résultats de l'analyse retard des mesures } \\
\text { de cellules (Fig. } 16 \mathrm{~b} \text { ). } \\
\text { Results of delayed response analysis of cells } \\
\text { data (Fig. 16b). }\end{array}$} \\
\hline Cellule & $\alpha_{2}$ & $\begin{array}{c}\eta_{2} \\
\text { (jours) }\end{array}$ & $\begin{array}{l}\text { Diffusivité } \\
\left(10^{-5} \mathrm{~m}^{2} / \mathrm{s}\right)\end{array}$ \\
\hline \multicolumn{4}{|c|}{ Niveau 488,50 (interface barrage/fondation) } \\
\hline $\begin{array}{l}\mathrm{C}_{1} \\
\mathrm{C}_{2} \\
\mathrm{C}_{3}\end{array}$ & $\begin{array}{l}0,44 \\
0,28 \\
0,07\end{array}$ & $\begin{array}{l}0 \\
0 \\
0\end{array}$ & $\overline{-}$ \\
\hline \multicolumn{4}{|c|}{ Niveau 497 NGF (corps du barrage) } \\
\hline $\begin{array}{l}\text { C4 } \\
\text { C5 } \\
\text { C6 }\end{array}$ & $\begin{array}{l}0,25 \\
0,21 \\
0,06\end{array}$ & $\begin{array}{l}35 \\
35 \\
43\end{array}$ & $\begin{array}{l}1,5 \\
2,0 \\
1,8\end{array}$ \\
\hline
\end{tabular}
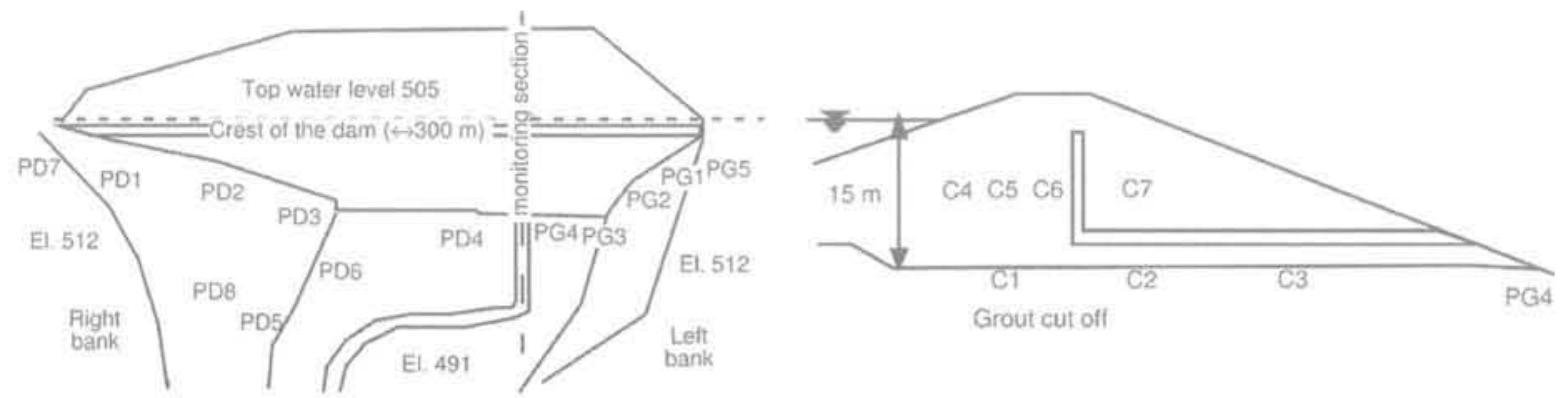

FIG.16 Vue en plan et implantation des piézomètres, coupe amont/aval du barrage dans l'axe ausculté et implantation des cellules de pression interstitielle.

Location map of piezometers in the plan view of the dam site, location map of the cells in the cross section. 
TABLEAUV Résultats de l'analyse retard des mesures de piézomètres (Fig. 16a).

Results of delayed response analysis of piezometer data (Fig. 16a).

\begin{tabular}{c|c|c|c|c|c|c|} 
Piézomètre & $\begin{array}{c}\text { Part } \\
\text { explicative } \\
\text { retenue }\end{array}$ & $\begin{array}{c}\text { Part } \\
\text { explicative } \\
\text { pluie }\end{array}$ & $\alpha_{2}$ & $\begin{array}{c}\eta_{Z} \\
\text { (jours) }\end{array}$ & $\begin{array}{c}\alpha_{0} \\
\text { (jours) }\end{array}$ & $\eta_{Q}$ \\
(jours)
\end{tabular}

Rive droite

\begin{tabular}{|c|c|c|c|c|c|c|}
\hline $\begin{array}{l}\text { PD1 } \\
\text { PD2 } \\
\text { PD3 } \\
\text { PD4 } \\
\text { PD5 } \\
\text { PD6 } \\
\text { PD7 } \\
\text { PD8 }\end{array}$ & $\begin{array}{l}87 \% \\
53 \% \\
21 \% \\
17 \% \\
12 \% \\
24 \% \\
86 \% \\
21 \%\end{array}$ & $\begin{array}{r}6 \% \\
6 \% \\
6 \% \\
45 \% \\
54 \% \\
46 \% \\
10 \% \\
49 \%\end{array}$ & $\begin{array}{l}0,77 \\
0,33 \\
0,21 \\
0,04 \\
0,05 \\
0,09 \\
0,85 \\
0,20\end{array}$ & $\begin{array}{l}5 \\
4 \\
3 \\
0 \\
3 \\
0 \\
7 \\
30\end{array}$ & $\begin{array}{r}77 \\
33 \\
24 \\
54 \\
76 \\
106 \\
156 \\
198\end{array}$ & $\begin{array}{l}39 \\
19 \\
13 \\
52 \\
34 \\
54 \\
66 \\
65\end{array}$ \\
\hline \multicolumn{7}{|c|}{ Rive gauche } \\
\hline $\begin{array}{l}\text { PG1 } \\
\text { PG2 } \\
\text { PG3 } \\
\text { PG4 } \\
\text { PG5 }\end{array}$ & $\begin{array}{l}88 \% \\
73 \% \\
23 \% \\
16 \% \\
32 \%\end{array}$ & $\begin{array}{r}6 \% \\
9 \% \\
36 \% \\
49 \% \\
31 \%\end{array}$ & $\begin{array}{l}1,00 \\
0,30 \\
0,07 \\
0,05 \\
0,71\end{array}$ & $\begin{array}{r}3 \\
5 \\
3 \\
11 \\
3\end{array}$ & $\begin{array}{r}109 \\
34 \\
39 \\
54 \\
608\end{array}$ & $\begin{array}{l}63 \\
16 \\
22 \\
40 \\
83\end{array}$ \\
\hline
\end{tabular}

cées de manière significative par la pluie. L'influence de la retenue est instantanée pour les trois cellules situées à l'interface avec la fondation, et retardée pour les trois cellules situées dans le corps du barrage, dans la zone de marnage. Le rabattement de la nappe phréatique est mis en évidence par la décroissance de $\alpha$, de l'amont vers l'aval.

Le tableau V donne les résultats des analyses des treize piézomètres. L influence de la retenue est peu retardée, sauf pour le piézomètre PD8 qui doit être interprété à partir de la connaissance de l'ouvrage. Le bon fonctionnement du voile d'étanchéité est illustré par la décroissance de $\alpha$, des rives vers le fond de vallée. Son contournement est illustré par des valeurs $\alpha_{2}$ de $\alpha$, proche d'un en haut des rives. Les piézomètres situés en fond de vallée sont fortement influencés par la pluie (PD4, 5, 6 et PG3, 4), contrairement à ceux situés sur les pentes (PD1, 2, 3 et PG1, 2), où la pluie ruisselle plus qu'elle ne s'infiltre. Le comportement du piézomètre PG5 est singulier : influence modérée de la retenue et forte influence de la pluie. Ceci peut être imputable à la présence d'une nappe de coteau détectée lors de la construction du barrage.

\section{6}

\section{Conclusion}

La littérature ne fait pas état d'une méthode opérationnelle qui permettrait d'analyser efficacement les pressions interstitielles mesurées sur les barrages, en fonction du niveau de la retenue et de la pluie. Les méthodes classiques d'analyse des séries chronologiques de type ARMA (Auto Regressive Moving Average), bien que d'usage courant dans d'autres domaines, sont rarement utilisées pour analyser les mesures d'auscultation de barrage. Nous proposons une méthode basée sur la réponse impulsionnelle (Impulse-Response-Function ou IRF). Le cas d'un retard exponentiel (exponential-IRF) est développé. Ce modèle est pertinent pour des écoulements à diffusivité peu variable, sous des sollicitations à variations lentes vis-àvis du temps caractéristique de diffusion. Il reproduit quelques aspects essentiels de l'effet différé : dissipation, accommodation (retard et amortissement) sous sollicitation cyclique, évolution fonction de l'histoire des sollicitations.

L'analyse des mesures d'auscultation de trois barrages montre que tous les instruments, cellules à cordes vibrantes dans le corps du barrage et piézomètres auscultant le corps du barrage, la fondation, les appuis et les rives sont a priori susceptibles d'exhiber un effet retard vis-à-vis du niveau de la retenue. Les piézomètres auscultant les rives peuvent être significativement influencés par la pluie. Les temps caractéristiques de diffusion sont de l'ordre de quelques jours à quelques mois.

L'IRF est intimement liée à la fonction de Green du problème aux limites de diffusion linéaire associé. Ceci permet de donner un sens mécanique au modèle IRF à partir des moments temporels. Il est en particulier possible d'estimer quelques valeurs de diffusivité hydraulique du corps du barrage. Elles sont de l'ordre de $10^{-5}$ à $10^{-3} \mathrm{~m}^{2} / \mathrm{s}$.

Le modèle exponential-IRF conduit une équation de récurrence permettant une mise en œuvre numérique simple des produits de convolution. Ce modèle ne nécessite pas la connaissance de l'histoire des sollicitations antérieures à la période d'analyse. La formulation discrète en temps est assimilable à un modèle de type ARMA $(1, q)$ où $q$ est le degré d'interpolation de la sollicitation. Ceci ouvre des perspectives quant à la possibilité d'utiliser des méthodes modernes pour analyser les mesures d'auscultation de barrages. 
Beck J.V., Cole K.D., Haii-Sheikh A. L.itkouhi B. - Heat Conduction Using Green's Functions. Hemisphere, Washington, DC, 1992.

Bonelli S., Félix H., Tourment R. - Interprétation des mesures d'auscultation des barrages par régression linéaire multiple HST. Fiabilité des matériaux et des structures. Hermès, 1998, p. 188-198.

Carrère A., Colson M., Goguel B., Noret C - -Modelling: a means of assisting interpretation of readings m, XXth International Congress on Large Dams, Beijing. vol. III, ICOLD, 2000, p. 1005-1037.

Crépon O., Lino M. - "An analytical approach to monitoring n. International Water Power \& Dam Construction. June. 1999, p. 52-54.

Fanelli M., Giuseppetti G., Mazza G. "Analysis of the phenomenon of time drift in the observational data of dam behaviour B. XXth International Congress on Large Dams, Beijing, vol. III, ICOLD, 2000, p. 1083-1098.

Faure P., Robin M. - Eléments d'automatique. Dunod, 1984, 448 p.

Ferry S. Willm G. - "Méthodes d'analyse et de surveillance des déplacements observés par le moyen de pendules dans les barrages x. VIth International Congress on Large Dams, New York, vol. II, ICOLD, 1958, p. 1179-1201.

Forbes J.D. - « Account of some experiments on the temperature of the earth at different depths and in different soils near Edinburgh w. Transactions of The Royal Society of Edinburgh, vol. 16, 1846, p. $189-236$.

Guedes Q.M., Coelho P.S.M. - "Statistical behaviour model of dams 3. XVth International Congress on Large Dams, Lausanne, vol. 1, ICOLD, 1985, p. 319-334

Kjaernsli B. Kvale G., Lunde J., BaadeMathiesen J. - « Design, construction. control and performance of the Svartevann earth-rockfill dam v. XIVth International Congress on Large Dams, Rio de Janeiro, vol. IV ICOLD, 1982 , p. 319-349

Lugiez F., Beaujoint N., Hardy X. - «L'auscultation des barrages en exploitation au service de la production hydraulique d’électricité de France, des principes aux résultats \%. Xth International Congress on Large Dams. Montréal, vol. III. ICOLD, 1970, p. 577-600.

Melnikov Yu. A. - "An alternative construction of Green's functions for the two-dimensional heat equation s. Engineering Analysis with Boundary Elements, $n^{\circ} 24,2000, p, 467-475$

Myrvoll F, Larsen S., Sande A., Romsol N.B. - t Field instrumentation and performance observations for the Vatnedalsvatn dams *. XVth International Congress on Large Dams, vol. I, Lausanne, ICOLD, 1985, p. 1039-1069.

Morse P. Feshbach H.- Methods of Theoretical Physics. Parts I and II. New York. McGraw-Hill, 1953.

Owen J.S., Eccles B.J., Choo B.S., Woodings M.A. - "The application of autoregressive time series modelling for the time-frequency analysis of civil engineering structures ${ }^{2}$. Engineering Structures, n023, 2001, p. 521-536

Poupart M. - $\alpha$ Contrôle de la sécurité par l'auscultation, apport de la télémesure e des méthodes de dépouillement des mesures x. XVIIIth International Congress on Large Dams, Durban, vol. V, ICOLD, 1994. p. 74-79.

Roach G.F. - Green's functions, introductory theory with applications. Van Nostrand Reinhold company, 1970, 280 p.

Roîtenberg LN. - Théorie du contrôle automatique. MIR, Moscou, 1974, 496 p.

Saint-Arnaud G - a The high pore pres. sures within embankment dams : ar unsaturated soil approach $n$. Canadian Geotechnical Journal, vol. 32, n² 6. 1995. p. $892-898$.

Santamaria J.C. Fratta D. - introduction to cliscrete signals and inverse problems in civil engineering. ASCE Press, Virginia, 1998. 328 p.

Silva Gomes A.F. Silva Matos D, - «Quantitative analysis of dam monitoring results, state of the art, applications and prospects 》1. XVth International Congress on Large Dams, Lausanne, vol. I. ICOLD. 1985, p. 749-761.

Willm G., Beaujoint N. - u Les méthodes de surveillance des barrages au service de a production hydraulique d'Electricite de France, problèmes anciens et solutions nouvelles w. IXth International Congress on Large Dams, Istanbul, vol. III, ICOLD. 1967, p. 529-550.

Windisch E. Høeg K. - «Pore pressure in the till core of Oddatjorn dam n. 53rd Canadian Geotechnical Conference. Montreal. 2000. p. 231-238.

Young P. - "Data-based mechanistic modelling of environmental, ecological. economic and engineering systems $\mathrm{s}$. Environmental Modelling \& Software. n²13, 1998, p. 105-122. 